\title{
Cervical Cancer Screening Postpandemic: Self-Sampling Opportunities to Accelerate the Elimination of Cervical Cancer
}

\author{
Taja Lozar ${ }^{1-3}$ \\ Rahul Nagvekar ${ }^{4}$ \\ Charles Rohrer $\mathbb{D D}^{5}$ \\ Racheal Shamiso Dube \\ Mandishora ${ }^{6,7}$ \\ Urska Ivanus $\mathbb{I D}^{3,8,9}$ \\ Megan Burke Fitzpatrick (D) ${ }^{1,5}$
}

'University of Wisconsin School of Medicine and Public Health, Madison, WI, USA; ${ }^{2}$ University of Wisconsin Carbone Cancer Center, University of Wisconsin School of Medicine \& Public Health, Madison, WI, USA; ${ }^{3}$ University of Ljubljana, Ljubljana, Slovenia; ${ }^{4}$ Department of Genetics, Stanford University, Stanford, CA, USA; ${ }^{5}$ Department of Pathology and Laboratory Medicine, University of Wisconsin School of Medicine and Public Health, Madison, WI, USA; ${ }^{6}$ University of Zimbabwe College of Health Sciences, Department of Medical Microbiology, Harare, Zimbabwe; ${ }^{7}$ Early Detection, Prevention and Infections Group, International Agency for Research on Cancer, Lyon, France; ${ }^{8}$ National Cervical Cancer Screening Programme and Registry ZORA, Epidemiology and Cancer Registry, Institute of Oncology Ljubljana, Ljubljana, Slovenia; ${ }^{9}$ Association of Slovenian Cancer Societies, Ljubljana, 1000, Slovenia
Correspondence: Megan Burke Fitzpatrick University of Wisconsin-Madison School of Medicine and Public Health, MC 8550, 600 Highland Ave, Madison, WI, 53792-8550, USA

Tel + I $608262-5922$

Fax +| 608 265-330|

Email mbfitzpatric@wisc.edu

\begin{abstract}
The persisting burden of cervical cancer in underserved populations and lowresource regions worldwide, worsened by the onset of the COVID-19 pandemic, requires proactive strategies and expanded screening options to maintain and improve screening coverage and its effects on incidence and mortality from cervical cancer. Self-sampling as a screening strategy has unique advantages from both a public health and individual patient perspective. Some of the barriers to screening can be mitigated by self-sampling, and resources can be better allocated to patients at the highest risk of developing cervical cancer. This review summarizes the implementation options for self-sampling and associated challenges, evidence in support of self-sampling, the available devices, and opportunities for expansion beyond human papillomavirus testing.
\end{abstract}

Keywords: self-sampling, self-collection, HPV testing, cervical cancer screening, COVID19

\section{Introduction}

Cervical cancer is the fourth-most common cancer in women worldwide, primarily affecting middle-aged women, particularly in low- and middle-income countries (LMICs). ${ }^{1}$ Despite the success of screening programs aimed at detection of precancerous and early cancerous lesions, ${ }^{2,3}$ limited access to and insufficient uptake of screening remain persistent barriers to cervical cancer elimination. ${ }^{4}$ Women who are not screened regularly or have never been screened have higher cervical cancer incidence and are diagnosed with more advanced disease with poor prognosis. ${ }^{5-7}$ When precancerous cervical lesions are detected early, there is nearly $100 \% 5$-year survival, but if caught at an advanced stage, 5-year survival drops to $17 \%{ }^{4,8}$ Early detection and treatment of cervical neoplasia is critical, and various options for screening and prevention exist.

Since human papillomavirus (HPV) infection is known to be the primary cause of cervical cancer, high-risk HPV (hrHPV) testing is frequently recommended in guidelines for early detection of cervical precancer and cancer. ${ }^{9-11}$ Commercially available HPV-testing platforms have been developed to detect and report only the hrHPV infections that are clinically relevant (eg, using cervical intraepithelial neoplasia 2 [CIN2] as the outcome of interest). ${ }^{12}$ Therefore, existing screening algorithms that incorporate HPV testing are based on these cutoffs for clinical sensitivity and are updated with approval of new testing platforms used for 
screening. While analytical accuracy for the detection of individual HPV genotypes is part of the validation of the assays, clinical accuracy is used as the outcome for most of the population-based studies presented herein.

Testing for hrHPV offers several advantages over cervical cytology alone, including higher sensitivity for detecting cervical precancer and the option of detection from self-collected samples with similar clinical accuracy as clinician-collected samples. ${ }^{9,10,13}$ Also, self-sampling does not require women to undergo a pelvic exam and can be performed without the presence of a trained clinician, which addresses common barriers to attendance in screening, including convenience and concerns of privacy and modesty. Furthermore, self-sampling is a method to reach never- or underscreened women. ${ }^{14,15}$ Self-sampling offers a unique opportunity for expansion to hard-to-reach populations, ${ }^{16,17}$ and is one of the most feasible strategies for mid- and postpandemic catch-up screening. With these advantages, self-sampling will in the future play an important part in improving the global coverage of cervical cancer screening with highly accurate HPV-testing, which - followed by treatment of detected high-grade cervical lesions - is a prerequisite to the global goal of cervical cancer elimination, together with HPV vaccination. ${ }^{18}$

In this review, we provide a summary of the implementation options for self-sampling, evidence in support of self-sampling, and the available devices. We also review existing evidence on cytological examination of self-collected samples and emerging molecular testing, and outline opportunities for product development.

\section{Profound Impact of the COVID-I 9 Pandemic on Cervical Cancer Screening}

The COVID-19 pandemic has had an enormous impact on cervical cancer screening. More than half of the $155 \mathrm{~min}-$ istries of health surveyed by the World Health Organization (WHO) postponed public screening programs in the spring of 2020 and reported disrupted cancer-treatment services. ${ }^{19}$ Among the most common reasons for reduced screening services, countries reported cancellation of planned visits, decreased public transportation availability, lack of staff, and shortages of medicines, diagnostics, and technologies. ${ }^{19}$ In a recent report, $63 \%$ of the 57 surveyed HPV reference and screening laboratories in both high- and low- to middle-income countries reported temporary suspensions of routine cervical cancer screening due to COVID-19. ${ }^{17}$

The magnitude of these disruptions in cervical cancer screenings during lockdowns in the spring of 2020 are emerging from countries with opportunistic and organized screening. ${ }^{20-22}$ In June 2020, cervical cancer screenings in the US were $35 \%$ lower than their pre-COVID-19 levels. Between March 15 and June 16, a deficit of $67 \%$ in cervical cancer screenings was noted relative to the number of screenings that would be expected based on the historical average. ${ }^{20}$ The suspension of inessential clinical services, including suspension of population-level screening programs, has impacted reports of cancer diagnoses worldwide, ${ }^{21,23-29}$ resulting in a decrease in overall cancer diagnoses compared to prepandemic data. In Slovenia, a European country with low cervical cancer burden as a result of a highly successful cervical cancer-screening program, a 2-month screening lockdown resulted in a $92 \%$ decrease in screenings and a $32 \%$ decrease in the diagnosis of high-grade lesions $\left(\mathrm{CIN}^{+}\right){ }^{21}$ This decrease in highgrade diagnoses persisted through the 5-month screening scaling-up phase, and was most notable in women aged 30-39 years (19\%), outlining a new vulnerable group within the program. ${ }^{21}$ The number of cancer screenings has since begun to rise, but has not yet reached previously expected levels. Failure to detect and treat precancerous lesions in settings in which cervical cancer had been successfully controlled by screening may have at least a temporary impact on progress toward decreasing the cervical cancer burden worldwide.

Barriers to screening disproportionately impact socioeconomically disadvantaged and minority women worldwide. $^{4,30,31}$ As a result, racial, sexual, and ethnic minorities and socioeconomically disadvantaged groups have higher incidence and rates of cancer-related deaths and lower rates of participation in guideline-based screening and treatment. ${ }^{32,33}$ Age, psychosocial issues, marital status, major life events, and competing interests (such as education, work, and childbirth) are important determinants of attendance at cervical cancer screening, and the COVID-19 pandemic may have exacerbated the effect of these determinants on screening coverage. ${ }^{34,35}$ After incorporation of updated screening recommendations, including hrHPV testing in 2012, an expected decrease in screening frequency among American women aged 30-65 years was observed, but an even more concerning drop in screening among women 21-29 years old. ${ }^{36}$ Australia reported that participants aged $>60$ years had had fewer HPV tests 
during COVID-19, and there was no bounce-back effect observed in this population even after the easing of pandemic-related restrictions. ${ }^{22}$

There are also unexpected opportunities for expansion of cervical cancer screening as a result of the COVID-19 pandemic. The pandemic has expanded diagnostic molecular testing technologies and infrastructure, supply chains, and overall molecular diagnostics activity at an unprecedented pace. As part of a survey conducted by Poljak et al, five companies currently offering clinically validated HPV and SARS-CoV2 tests (Roche Diagnostics, Indianapolis, IN, USA; Abbott Laboratories, Chicago, IL, USA; Cepheid, Sunnyvale, CA, USA; Becton Dickinson, Franklin Lakes, NJ, USA; and Hologic, Marlborough, MA, USA) released a statement about their operations during the pandemic and their current and postpandemic strategies for HPV tests. ${ }^{17}$ All companies noted increases in manufacturing capacity and investment in new supply chains, molecular and core laboratory solutions, and the associated expansion for molecular diagnostics, including the use of platforms acquired for COVID-19 testing to support HPV testing in the future. The availability of both the infrastructure and highly trained personnel currently working on COVID-testing could be allocated toward increased HPV molecular testing capacity. In addition, low-cost portable testing technologies ${ }^{37-39}$ that have been refurbished to address large-scale testing requirements of the pandemic could be repurposed to provide affordable screening assays for HPV testing, especially in lowresource settings.

\section{Self-Sampling Implementation and Dissemination Strategies Improving Coverage in Organized and Opportunistic Screening}

Well organized, population-based cervical cancer-screening programs have substantially decreased the incidence of and mortality from cervical cancer. ${ }^{2,3,14,15}$ The success of these programs is dependent on sufficient attendance for high-quality screening, as well as robust diagnostic, follow-up, and treatment services. In organized populationbased programs with high screening coverage, half or more of new cases are detected in nonattenders, and these cancers are more often diagnosed in advanced stages. $^{40-42}$
Worldwide, self-sampling for HPV testing has been proposed as a strategy to reach nonattenders of organized screening programs. ${ }^{10,43}$ A meta-analysis of reported effect sizes from 29 randomized clinical trials found that women were twice as likely to use cervical cancer-screening services through self-sampling than standard-of-care screening practices (response ratio [RR] 2.13). ${ }^{44}$ In their response to the WHO's 2020 call for cervical cancer elimination as a public health problem, a European task force considered incorporating self-sampling into organized screening programs to support the achievement of the WHO goal in Europe. ${ }^{18}$

In countries with opportunistic screening, such as Canada and the US, there are suboptimal participation rates in clinic-based screening ${ }^{31,49,50}$ and thus increasing interest in improving participation through self-sampling options. A recent study found that $72.7 \%$ of US women reported high willingness to use an HPV self-sample kit at home $^{51}$ which is consistent with other studies from the US. ${ }^{52-58}$ In the first randomized controlled trial evaluating the feasibility of mailing HPV self-sampling kits to underscreened US women, more than half the women chose to return a self-collected sample, rather than schedule an in-office screening. ${ }^{59}$ Canadian women living in rural Ontario who were overdue for cervical cancer screening were 3.7 times as likely to undergo screening with a self-sampling kit as those who received the standard-ofcare opportunistic screening. ${ }^{60}$ While HPV self-collection is not currently an FDA-approved cervical cancer screening strategy in the US or Canada, increasing evidence in support of self-collection and the effect of the pandemic on screening coverage might prompt future consideration.

In countries with organized screening programs in high-resource settings, research has focused on two approaches to offering self-collection depending on whether the self-collection device is readily available: "opt in" and "opt out." In both approaches, women collect a self-sample outside the clinic using a variety of sampling-kit types.

In the opt-out or mail-to-all approach, self-collection devices are mailed to women's homes without them taking the initiative. Recent systematic reviews and meta-analyses have shown that this approach can significantly increase attendance and detection of high-grade cervical lesions compared to invitation or reminder letters. ${ }^{9,10,45}$ The most recent meta-analysis of randomized control trials showed a doubling of the likelihood of attending screening compared with controls (RR 2.27). ${ }^{44}$ 
An alternative invitation scenario is the opt-in approach, in which women request a self-collection kit via email, text message, phone, website, or mail. The kit is mailed to their desired address or they pick it up at a pharmacy or clinic. This approach is more economical, especially considering the costs of implementation on a national level. ${ }^{9,10,46}$ However, studies evaluating this approach have shown variable response rates. ${ }^{9,46}$ Response rates in early randomized opt-in studies were comparable to control groups (RR 0.97, 1.22, and 1.28) in published meta-analyses, ${ }^{9,10,44}$ and much lower in opt-out groups (RR 1.22 vs 2.33). ${ }^{9}$ Recent randomized studies have shown the opt-in approach can generate a high response when compared to mailing a reminder letter (RR 1.8), ${ }^{46}$ although lower than response rates to the opt-out approach (RR 2). ${ }^{46-48}$

Door-to-door or community outreach is an approach to self-sampling that has primarily been investigated in lowresource settings or for reaching underscreened populations in high-resource settings. In this approach, an educated health worker, such as a nurse or a community health worker visits participants' homes to educate participants on cervical cancer and screening, offer a self-sampling HPV test kit to the participant, and collect the kit for lab testing. Metaanalysis of five randomized clinical trials using this approach showed that women were almost three times as likely to participate in cervical cancer screening (RR 2.37). ${ }^{44}$ A prospective cohort study on community outreach in the rural Mississippi Delta in the US found that $80 \%$ of underscreened women responded to an offer of the HPV self-sampling kit and cervical cancer education compared to $40.5 \%$ who responded to a voucher for a free Pap test at the local clinic. ${ }^{61}$ Increasing social inequality related to the pandemic, such as loss of financial stability and health insurance, lack of transportation, and lack of childcare, is likely to primarily affect already underserved populations, and approaches such as this could be considered to reach these populations.

The key challenge in implementing self-sampling in any setting is ensuring follow-up and treatment for women with positive tests ${ }^{62}$ and assuring adequate training for and supervision of these services. ${ }^{63}$ In low-resource settings, attendance at follow-up care is $14 \%-95 \%,{ }^{64}$ and in high-resource settings $41 \%-100 \%{ }^{47,65-67}$ after an HPVpositive self-sampling result.

The most recent meta-analysis of 20 reports of attendance at follow-up care among women with hrHPV- positive self-samples found that $80.5 \%$ had a follow-up examination. This was influenced by triage policy: studies with direct referral had higher attendance at follow-up care than studies with a triage policy. ${ }^{9}$ In low-resource settings, follow-up rates are improved by engaging community health workers, ${ }^{68,69}$ ensuring rapid (same day) availability of test results, $^{70}$ and offering community mobile treatment. ${ }^{71}$ In high-resource settings, follow-up in selfsampling is relatively high, but can still be improved through prescheduled appointments, personal contacts via telephone with women who did not attend, prescheduled reminders, ${ }^{46}$ reminder letters to HPV-positive women, and direct contact with a physician to explain the test results and their consequences. ${ }^{72}$

\section{Improving Access to Cervical Cancer Screening in Resource-Limited Regions}

Resource-limited regions have the highest burden of cervical cancer incidence and mortality worldwide: $90 \%$ of deaths caused by cervical cancer are recorded in LMICs. ${ }^{73}$ This disparity can be attributed to a lack of organized screening programs and limited access to resources, health-care facilities, and providers. ${ }^{74,75}$ For example, in Uganda and Zimbabwe, only 5\%-10\% of women in rural areas have been screened for cervical cancer. ${ }^{69,76,77}$

In resource-limited regions, the infrastructure to conduct organized screening with a Pap test is often not available, so visual inspection with acetic acid (VIAC; with/without cervicography) is used as an alternative cost-effective screening strategy. ${ }^{78}$ VIAC involves trained nurses that examine the cervix via a speculum examination after application of acetic acid (vinegar) to identify potentially precancerous lesions. A screen-and-treat approach incorporating non-cytology-based screening with HPV testing or VIAC followed by immediate treatment using cryotherapy or thermal ablation has also been implemented in many settings successfully. This approach avoids the need for the complex health-care infrastructure required for a robust cytology-based and callback system.

Program evaluations have found that overtreatment rates with VIAC can be high and result in many women undergoing unnecessary treatments. Overtreatment can lead to future pregnancy complications in women of reproductive age. ${ }^{79}$ Furthermore, access to VIAC in rural areas is limited, since there are few screening facilities that offer cancer screening and overburdened health-care workers may not be available for screening. ${ }^{62,80,81}$ 
An alternative method for improving access to cervical cancer screening is through self-collected HPV testing. Self-sampling offers a unique opportunity to bring robust and high-sensitivity cervical cancer screening to resourcelimited rural communities with limited transportation options, where women usually must travel to distant health facilities. Furthermore, self-sampling can encourage screening participation by respecting women's privacy, removing the need for a pelvic exam and avoiding feelings of shame and fear related to exposure of private parts, especially in the presence of a male health-care worker, thus removing the need for spousal permission and fear of social marginalization. ${ }^{82,83}$ In addition, the screening interval following a negative result can be extended and screening initiated at an older age, reducing the lifetime screening visits required. ${ }^{84}$ There is increasing evidence that self-collected HPV testing is more cost-efficient than other screening strategies in developing countries. ${ }^{84,85}$

Randomized trials in Africa and Latin America have used community campaigns or engaged community health workers to deliver self-sampling kits directly to women's homes or workplaces (door to door), and have shown improved response rates when compared to reminder or invitation letters (RR 2.58 for community campaigns and 2.01 for door to door). ${ }^{9}$ Community-based self-sampling in Ghana and Kenya has shown higher participation rates than hospital-based self-sampling. ${ }^{86,87}$ Community-based screening using HPV testing on self-samples in rural Zimbabwe led by community health workers showed profound increases in participation in cervical cancer screening, with an overall response rate of $82 \%$ compared to a baseline screening rate of $5 \% .{ }^{69,88,89}$ This approach offers the possibility of reaching women living in remote locations with long travel times to health-care centers.

Self-sampling has generally been well accepted among African women, ${ }^{64,83}$ and has shown comparable sensitivity and specificity to detect clinically relevant infections when compared to clinician-collected samples. ${ }^{64}$ Pooled analysis of 38 studies in LMICs showed most participants found self-sampling easy to perform (75\%-97\%, 18 studies), painless $(60 \%-90 \%$, nine studies), and preferred to provider-collected sampling $(57 \%-100 \%, 14$ studies $) .{ }^{64}$ However, some studies in LMICs found women feared hurting themselves ${ }^{90}$ and expressed the need for assistance with self-sampling. ${ }^{90,91}$

Although introducing self-sampling in low-resource regions is important to increase the early detection and treatment of precancerous cervical lesions and meet screening targets, there are potential challenges that need to be addressed for optimal outcomes. The first is education and support to increase women's confidence in performing self-sampling correctly. The success of self-sampling is most impactful when women accept and prefer the method. Secondly, organized screening in high-income settings is made possible by efficient postal services and immediate communication through mobile phones, which may not be feasible in low-resource settings, due to inconsistent power supply and potential lack of privacy if their partners are the primary users of a shared mobile phone. Community-based approaches using and expanding on existing infrastructure and emphasizing education and linkage to follow-up care are critical when considering integration of self-sampling as a component of a cervical cancer-screening strategy in lowresource settings.

\section{Diagnostic Accuracy: Latest Evidence}

Self-sampling shows good agreement for detection of hrHPV, with analytical sensitivity for detection of hrHPV of $91.4 \%-96.8 \%$ in studies comparing self-samples with clinical collected samples. ${ }^{13}$ However, as outlined by Meijer et al, hrHPV-test requirements for primary cervical cancer screening should be based on clinical accuracy criteria (detection of clinically relevant infections), rather than analytical accuracy criteria (detection of hrHPV infection). The key issue for hrHPV testing in primary cervical cancer screening is to detect clinically relevant infections that are associated with or develop into CIN2 or higher and differentiate them from transient HPV infections to avoid redundant or excessive follow-up. ${ }^{12,92}$

Before widespread adoption of PCR-based technology, signal-based amplification assays for the detection of hrHPV were the primary testing platform used for screening. Signal-based amplification detection of hrHPV shows lower clinical sensitivity using self-samples than cliniciancollected cervical samples for detection of $\mathrm{CIN} 2^{+} .{ }^{-}$ However, with modern hrHPV assays based on PCR technology, self-samples have clinical sensitivity comparable to clinician-collected samples $(96 \%)$ for detection of $\mathrm{CIN} 2^{+}$.

The improved diagnostic accuracy of self-sampling using PCR-based HPV-testing assays has led to some countries, such as Australia, the Netherlands, and Denmark, ${ }^{93,94}$ to institute a self-collection option as part of their organized national screening programs to reach under- or never- 
screened women and those who decline a clinician-collected sample. Other European countries with organized screening programs have been investigating the feasibility and cost-effectiveness of implementing a self-collection option following the recommendations outlined in the European guidelines for quality assurance in cervical cancer screening. ${ }^{46,48,95-97}$

Emerging data support exploring the potential of selfsampling for primary cervical cancer screening, ${ }^{98}$ and suggest that benefits of increased participation outweigh the worst case possible of $2 \%$ relative loss of sensitivity when testing on self-collected samples. ${ }^{99}$

\section{Types of Devices for HPV Self-Sampling}

Studies of self-sampling in both rural and urban settings have employed a wide variety of devices: cervicovaginal brushes, ${ }^{46,72,100-102}$ vaginal swabs, ${ }^{46,61,103,104}$ tampon-like devices, ${ }^{105}$ and lavage devices. ${ }^{46,102,106-108}$ All types of devices are based on collecting exfoliated cells of the cervicovaginal canal for subsequent HPV DNA detection. A comprehensive description of widely investigated selfsampling devices is presented in Figure 1.

The first studies of self-sampling for cervical cancer screening were performed using standard cytobrushes that are used for clinician-based cervical sampling, or synthetic and cotton swabs used for other diagnostic purposes. In 2005, a meta-analysis by Ogilvie et al reported pooled overall sensitivity of $74 \%$ and specificity of $88 \%$ for HPV DNA detection across studies that used cytobrushes or Dacron/cotton swabs when compared to clinician-collected samples. ${ }^{109}$ Most of these studies used signal amplification-based HPV assays (such as Hybrid Capture), which we now know are associated with lower sensitivity than current PCR-based assays. Self-sampling with a cytobrush, ${ }^{110,111}$ as well as a synthetic (Dacron) swab, ${ }^{112}$ was well accepted and considered easy to perform among participants in clinic-based studies.

Once devices specifically designed for cervicovaginal self-sampling were introduced on the market, several studies provided insights into women's preferences. Women prefer devices (brush or swab-based) that are smaller and colorful in appearance. ${ }^{55,56,113}$ In a recent study in the US, women were more willing to use devices that closely resemble a basic swab than three other swab- and brushbased devices. ${ }^{51}$ Interestingly, this study also found women's willingness to use a specific brush device varied by sexual orientation: sexual minority women were more willing to use the HerSwab device than heterosexual women.

In a meta-analysis by Arbyn et al, all types of swab-, brush-, and lavage-based devices were associated with high sensitivity when used with a PCR-based HPV assay. Brush- and swab-based devices were slightly more sensitive $(98 \%, 95 \%$ CI $0.93-1.03)$ than lavage-based devices (95\%, 95\% CI 0.87-1.04; Figure 1). ${ }^{9}$ In a Norwegian study, samples collected using a brush-based device had similar sensitivity for $\mathrm{CIN}^{+}$to clinician-collected samples, whereas samples collected using a swab-based device did not. ${ }^{114}$ There are reports suggesting a preference for brush-based devices compared to swab- or lavage-based devices, ${ }^{114,115}$ but these are not consistent. ${ }^{102}$

The type of HPV self-sampling device may play an important role in women's acceptability of a screening strategy. Studies in the Netherlands and Finland found offering a brush-based device is comparable to offering a lavage-based one in terms of participation and acceptance by women; however, brush-based devices had slightly higher participation in both studies $(23.8 \%$ vs $21.7 \%$ in the Finnish study and $34.6 \%$ vs $31.9 \%$ in the Dutch study). ${ }^{96,102}$ There were no observed differences in hrHPV-positivity rate or sample-inadequacy rate. ${ }^{102}$

\section{First-Void Urine Collection as an Alternative Self-Sampling Strategy}

As an alternative for women reluctant to undergo clinician-based cervical cancer screening and vaginal self-sampling, home-based first-void urine collection for hrHPV testing may be valuable. Urine samples are a cheap, noninvasive, and easy-to-collect approach to self-sampling for HPV testing ${ }^{116}$ and have been highly accepted when offered in clinics and more recently at home. ${ }^{117-120}$ However, when compared to vaginal self-sampling, the diagnostic accuracy is somewhat lower. In 2014, a metaanalysis by Pathak et al found pooled sensitivity of $77 \%$ and specificity of $88 \%$ for hrHPV detection in urine samples compared with clinician-collected cervical samples. ${ }^{121}$ Similar positivity rates and sensitivity for $\mathrm{CIN}^{+}$and $\mathrm{CIN}^{+}$for urine and vaginal self-samples using dry and wet swabs were reported in another British clinic-based study. ${ }^{119}$ Ørnskov et al found absolute sensitivity for $\mathrm{CIN}_{2}{ }^{+}$of urine and vaginal self-samples was comparable to cervical clinician-collected samples $(93 \%$ for urine samples and $96 \%$ for self-collected samples). ${ }^{122}$ 


\begin{tabular}{|c|c|c|c|}
\hline TYPE OF DEVICE & LAVAGE & BRUSH & SWAB \\
\hline \multicolumn{4}{|l|}{ APPEARANCE } \\
\hline DESCRIPTION & $\begin{array}{l}\text { The lavage-based device is pre- } \\
\text { filled with sterile saline which is } \\
\text { released into the vagina after } \\
\text { insertion. The saline then flows } \\
\text { back into the device, thus } \\
\text { exfoliating cells from the cervix } \\
\text { and vagina. }\end{array}$ & $\begin{array}{l}\text { The brush is attached to a plastic } \\
\text { applicator stick or stored inside a } \\
\text { plastic applicator. The brush is } \\
\text { inserted into the } \\
\text { vagina, pushed out of the plastic } \\
\text { applicator if needed and rotated } \\
\text { to collect the cells, then } \\
\text { removed and stored back into the } \\
\text { plastic applicator or removed } \\
\text { from the applicator stick to be } \\
\text { stored in a separate container. }\end{array}$ & $\begin{array}{l}\text { A swab device consists of a } \\
\text { plastic applicator stick with a dry } \\
\text { or flocked swab tip that a woman } \\
\text { inserts into the vagina without } \\
\text { touching the external genitalia } \\
\text { and rotates before removal. }\end{array}$ \\
\hline $\begin{array}{l}\text { SENSITIVITY TO DETECT } \\
\text { CIN2+/PCR assay }{ }^{12}\end{array}$ & $0.95(95 \% \mathrm{Cl} 0.87$ to 1.04$)$ & $0.98(95 \% \mathrm{Cl} 0.95$ to 1.02$)$ & $0.98(95 \% \mathrm{Cl} 0.93$ to 1.03$)$ \\
\hline $\begin{array}{l}\text { COMMERCIALLY AVAILABLE } \\
\text { DEVICES }\end{array}$ & $\begin{array}{l}\text { Delphi Screener (Rovers Medical } \\
\text { Devices B.V., Oss, The } \\
\text { Netherlands) }\end{array}$ & $\begin{array}{l}\text { Viba Brush, Evalyn Brush } \\
\text { (Rovers Medical Devices B.V., } \\
\text { Oss, The Netherlands) }\end{array}$ & $\begin{array}{l}\text { HerSwab (Eve Medical, Toronto, } \\
\text { Ontario, Canada), FLOQSwab } \\
\text { (Copan Diagnostics, Italy), Coari } \\
\text { (Kolplast, Sao Paulo, Brazil). } \\
\text { Qvintip (Approvix, Uppsala, } \\
\text { Sweden) }\end{array}$ \\
\hline
\end{tabular}

Figure I Review of the key types of self-sampling devices.

Abbreviation: $\mathrm{Cl}$, confidence interval.

Furthermore, a majority of studies of HPV testing on urine samples have noted lower hrHPV positivity than corresponding vaginal samples. ${ }^{118,120,123-125}$

Home-based urine collection is well accepted among Danish women, and a majority of them would choose urine testing for future screening. ${ }^{120}$ Rates of concerns about proper urine self-sample collection are $3 \%-20 \%$ of surveyed women. ${ }^{118,120}$ Large-scale participation studies of urine selfsampling are currently lacking. A first-void urine self-collection device has already been made commercially available. ${ }^{126}$

\section{Lessons in Women's Preferences}

Women participating in self-sampling studies have consistently reported positive attitudes toward the use of selfcollected samples for HPV testing ${ }^{127-130}$ and a preference for self-sampling to clinician-based sampling for future screening. ${ }^{96,102,130-134}$ Studies in nonattenders showed that women find self-sampling to be more convenient, less embarrassing, less uncomfortable, and less painful than clinician-based sampling. In Argentina, self-sampling is preferred, because it allows women to overcome barriers related to the health system (ie, long waiting times) without sacrificing time devoted to work/domestic responsibilities. $^{135}$ For women, the most appealing features of self-sampling are cost (free), convenience (home-based), and less anticipated discomfort than a Pap test. $^{132}$

Across many countries and age-groups, women are able to perform self-sampling with simple written instructions. ${ }^{132,136}$ A study on Australian women's attitudes toward self-sampling noted small kits that fit in mailboxes are preferred over having to collect a parcel at a post office. ${ }^{132}$ In Finland, a combination of interventions (reminder letter and then self-sampling kit) increased total participation from $63 \%$ (regular invitation) to $78 \%,{ }^{108}$ suggesting that an affordable self-sampling kit or device could be included with a reminder letter to screening nonattendees. ${ }^{43}$ A recent Brazilian study suggested that women were more likely to respond to screening if self-sampling were offered as an option. ${ }^{137}$ A randomized controlled trial in Belgium noted self-sampling was particularly well accepted among postmenopausal women compared to women aged $<50$ years (OR 6.4 vs 2.1). ${ }^{138}$

The most commonly raised concerns in participants to self-sampling are test accuracy and whether they are performing the procedure correctly. ${ }^{51,52,57,58,133,136,139-142}$ In the Dutch IMPROVE study, women reported significantly lower levels of shame, nervousness, discomfort, and pain 
during self-sampling than with clinician-based sampling; however, trust in correct sampling was higher during clinician-based sampling. ${ }^{130}$ A qualitative British study found the Muslim population was especially concerned about not performing the test correctly and would prefer to continue to be screened by a health professional. ${ }^{66}$ A preference for clinician-based screening (73\%) was also reported in a study of sexually active adolescents at an American urban teen health center undergoing both self-sampling and clinician-based sampling. This was mainly due to a lack of faith in performing the sampling correctly. ${ }^{143}$ Lastly, some qualitative studies in middle-income countries have reported that women fear hurting themselves when taking the self-sample. ${ }^{82,135}$ On the other hand, nearly $20 \%$ of women indicate that they do not have any concerns about using a self-sample. ${ }^{51}$ To address women's concerns, positive feedback from large-scale studies should be presented through public-awareness campaigns, including data showing that most women are able to successfully obtain an adequate sample. ${ }^{51,96,102,115}$

Implementation of self-sampling should be coupled with educational interventions that would raise awareness of the importance and impact of cervical cancer prevention in general, including HPV vaccination of children and cervical cancer screening of adult women. Educational interventions aimed at increasing awareness about HPV infection and cervical cancer risk are associated with higher acceptability of self-sampling. ${ }^{144}$ Taiwanese women who perceived their cervical cancer risk as high and had high-level of HPV-related knowledge were more likely to perform HPV self-sampling. ${ }^{145}$ Conversely, levels of HPV-related knowledge and perceived risk are low in underscreened women from low-income areas; however, they do perceive mailed self-sampling kits as trustworthy. ${ }^{146}$ In addition, the causal relationship between cervical cancer and sexually transmitted HPV infection can result in perceived sexual promiscuity by the community and feelings of shame and blame in women who test positive for HPV. ${ }^{147,148}$ Stigma associated with cervical cancer and HPV can influence women's willingness to undergo an HPV test and their interpretation of screening results. ${ }^{149,150}$ In some settings, there are pervasive misperceptions that the "type of women" who get cervical cancer are uneducated, sexually promiscuous, and cursed. ${ }^{77,89,151}$ Furthermore, HPV testing can raise potentially difficult issues related to trust and fidelity within marriages in cases of a positive test result. ${ }^{113}$ Due to the financial implications of testing and treatment, women in certain cultures often need to obtain spousal consent/approval to receive screening and care. ${ }^{77,151,152}$

\section{Self-Sampling Beyond HPV Testing Cytology}

While hrHPV self-sampling has shown comparable diagnostic agreement to clinician-collected samples, self-collected Pap smears are considered insufficiently sensitive using currently available collection devices. ${ }^{153}$ Few studies have compared the accuracy of cytology between self-samples and clinician-collected samples using different types of self-sample devices, demonstrating fair-moderate agreement and lower sensitivity on self-collected samples. ${ }^{153-158}$

In one of the earliest studies of self-collected Pap tests, ${ }^{154}$ sensitivity for self-collected cytology using a traditional cytobrush was significantly lower than clinician-sampled cytology (55\% vs $85 \%$ ); however, specificity for self-collected samples was higher ( $85 \%$ vs $73 \%$ ). Since that time, a variety of self-collection methods and commercial and noncommercial devices have been investigated, with varying results. When reported, relative sensitivities for $\mathrm{CIN}^{+}$were low and ranged between $33 \%$ and $75 \% .{ }^{159,160}$ Several studies have shown comparable or improved specificity of self-collected cytology compared to clinician-collected samples. ${ }^{155,157,159}$

In a recent prospective cohort study, Loopik et al showed reflex cytology is feasible on hrHPV-positive self-samples and could be considered as an additional triage test for immediate referral to decrease loss to follow-up in screening. ${ }^{161}$ The authors also noted a lack of endocervical, endometrial, and inflammatory cells, which made interpretation of the slides easier, but not necessarily as reliable.

Despite the challenges with self-Pap, the possible increased positive predictive value when combined with self-collected HPV testing may still provide promise. Combining hrHPV self-sampling with a self-Pap could improve access to screening while increasing the positive predictive value of an hrHPV-test result. Improved devices may improve the validity of the results, but the challenge of self-guided collection of the squamocolumnar junction continues to present a significant hurdle to incorporating self-Pap collection in cancer screening. The rapid pace of discovery of potential biomarkers predictive of high-grade cervical lesions from cytology fluid may offer additional predictive value in future, and these are briefly reviewed in the next section. 


\section{Emerging Biomarkers and Molecular Approaches: The Future of the "Liquid Biopsy"}

Since $<10 \%$ of acute HPV infections progress to highgrade lesions or invasive cancer, there is a need for appropriate tools to triage HPV infections. ${ }^{162}$ In high-income countries, women with either an abnormal Pap smear or histology-confirmed $\mathrm{CIN}_{2}{ }^{+}$are generally referred for follow-up or treatment, but these approaches are impractical in many low-resource settings, due to the labor and equipment required. ${ }^{62,163}$

Both self-collected cervicovaginal samples and firstvoid urine samples will likely not fulfil the high-quality cellularity standards required for morphological review. Molecular biomarker assays may represent an alternative for detecting cervical lesions of clinical concern. In future, many of these assays may be suitable to perform on selfcollected samples, and could offer additional specificity for detection of precancerous cervical lesions when combined with HPV screening. For example, high-throughput massively parallel-sequencing technologies, known as next-generation sequencing (NGS), could be applied in a "molecular Pap test" on exfoliated cells from a selfcollected sample. ${ }^{163}$ NGS-based components of this molecular Pap test might include:

- HPV genotyping. Detection of HPV types and variants, for which NGS is more sensitive than PCR, ${ }^{164}$ which could be useful in triage because variants of HPV types often exhibit differences in infectivity, duration of persistence, and/or oncogenicity. ${ }^{163}$ Infection with multiple HPV types, which occurs commonly, can be detected suitably with NGS and may be associated with reduced risk of cervical cancer. ${ }^{164}$

- Identification of HPV integration sites in the host genome using high-throughput viral integration detection (HIVID); tagging, enrichment, and NGS of HPV16 (TEN16); or other NGS-based assays utilizing customized HPV-specific probes. ${ }^{164,165} \mathrm{HPV}$ integration rates are positively correlated with CIN grade and can be a predictor of likely progression to cancer. ${ }^{164}$

- Host/viral gene methylation. Quantification of levels of DNA methylation, an epigenetic modification, at numerous $\mathrm{CpG}$ sites have been proposed as biomarkers for cervical precancer. ${ }^{166}$ Methylation levels of the HPV16 L1 and L2 genes can be used to identify HPV16-induced high-grade CIN with high diagnostic accuracy, ${ }^{166}$ and a recent meta-analysis of methylation at the human CADM1, MAL, MIR124-2, FAM19A4, POU4F3, EPB41L3, PAX1, and SOXI genes, as well as HPV16 L1 and L2, concluded that DNA methylation was significantly higher in $\mathrm{CIN} 2^{+}$ relative to $\leq \mathrm{CIN} 1 .{ }^{167}$ Additionally, methylation frequency at the promoters of multiple human genes is significantly higher in cervical cancer samples than controls. ${ }^{168}$ When considering performance on selfcollected samples, one study found that the methylation levels at PAX1, SOX1, and ZNF582 showed no significant differences between self-collected and clinician-collected samples in specificity or sensitivity as predictors of $\mathrm{CIN}^{+} .{ }^{169}$ However, HPV-methylation studies to date have been limited in their geographic spread and HPV types studied: most have focused on HPV $16,{ }^{170}$ and few have been conducted in Africa, ${ }^{168}$ despite sub-Saharan Africa having the highest incidence of cervical cancer worldwide. ${ }^{171}$ Further clinical studies with large cohorts are recommended to fully evaluate the performance of methylation assays as predictors of CIN $2^{+} .{ }^{172}$

The cost of NGS continues to decline dramatically, and it is predicted that NGS technologies will soon be suitable for widespread adoption in cervical cancer screening. ${ }^{164}$ Nonetheless, a recent systematic review by Onyango et al concluded that dual staining for the cell cycle proteins p16 and Ki67, which is not NGS-based, currently represents the most clinically useful biomarker assay to detect CIN2 ${ }^{+} \cdot{ }^{172}$ High p16 expression is known to be associated with persistent hrHPV infection, while Ki67 is a marker of cell proliferation associated with malignant tumors. Additionally, since p16 induces cell-cycle arrest under physiological conditions, coexpression of p16 and Ki67 is observed in cells with dysregulation of the cell cycle. ${ }^{173}$ Multiple studies have established dual p16-Ki67 staining as an appropriately sensitive and specific method for identifying HPV infections of clinical concern, ${ }^{174-177}$ and it has also been demonstrated that p16-Ki67 assays can be conducted accurately by minimally trained evaluators. ${ }^{178}$ Limitations include variability in specificity depending on patient age and the threshold number of $\mathrm{p} 16^{+}-\mathrm{Ki} 67^{+}$cells 
used to define a positive result. ${ }^{179}$ Toliman et al found dual p16-Ki67 staining had higher sensitivity in predicting high-grade disease on clinician-collected cervical specimens than self-collected vaginal specimens. ${ }^{180}$ Further studies are needed before recommending dual p16-Ki67 staining as a triage test for HPV-positive women, and it may be replaced by emerging molecular markers. ${ }^{181}$

Other emerging biomarkers that have been proposed to predict $\mathrm{CIN}_{2}{ }^{+}$include the HPV mRNAs E6 and E7 and various human microRNAs (miRNAs) and proteins, such as SCCAg, MCSF, and VEGF. When overexpressed, the protein products of HPV E6 and E7 inactivate human tumor suppressors, and testing for E6 and E7 appears to have diagnostic relevance in detecting $\mathrm{CIN} 2{ }^{+} .{ }^{182} \mathrm{HPV}$ mRNA assays, many of which detect E6 and E7, have been proposed as a tool for secondary cervical cancer screening after HPV DNA testing as primary screening. ${ }^{183}$ Pardini et al identified miR21 and miR29a as the most frequently upregulated and downregulated human miRNAs, respectively, in studies of invasive cervical cancer progression. ${ }^{184}$

\section{Cost-Effectiveness of Self-Sampling}

It is generally accepted that self-sampling is a cost-effective strategy, and there is ongoing investigation into optimal screening protocols. The largest reductions in monetary costs associated with self-sampling are the decrease in excess office-based exams for women testing HPV-negative, potential reductions in unnecessary colposcopy referrals, unnecessary treatments.

The cost-effectiveness of self-sampling is largely dependent on the implementation approach. The opt-in approach offers some economic and environmental advantages. However, using mathematical modeling on data from a randomized self-sampling trial in Norway, Burger et al found that the costs saved by an opt-in approach were relatively small (2\%) and overshadowed by other larger screening-related costs (eg, office-based exams, colposcopies). ${ }^{185}$ Targeted 5 -yearly self-sampling of women who did not respond to reminder letters or participate in organized screening have been found to reduce the lifetime risk of developing cervical cancer with an attractive cost-effectiveness profile: US\$29,630$\$ 29,420$ per quality-adjusted life year gained compared with no screening. However, the magnitude of the health benefit and optimal self-sampling strategy was dependent on the profile and behavior of self-sampling respondents (time since last screening, never screened). ${ }^{186} \mathrm{~A}$ cost- effectiveness analysis from Sweden showed HPV selfsampling using the opt-out approach led to more women being screened and histologically diagnosed $\mathrm{CIN} 2^{+}$at a lower cost than midwife-collected Pap smears. ${ }^{187}$

An important consideration when considering implementation of self-sampling as a screening strategy is avoiding overscreening or double-screening. Doublescreening is costly, and in the era of improved sensitivity it adds no benefit. In the Norwegian pilot study, $18 \%$ of women who did not have an hrHPV infection attended an office-based exam, despite being explicitly advised that they did not need further screening. ${ }^{115}$ A modeling study based on these data showed that eliminating potential overscreening practices was one of the most important cost-saving practices that a self-sampling screening program could undertake. ${ }^{186}$ In the Slovenian randomized pilot self-sampling trial, women in the opt-in arm could choose to order a self-sampling kit or schedule an in-office visit, while women in the opt-out arm had received selfsampling kits and had free access to screening at a clinic. In the opt-in arm, only $1.8 \%$ of women performed both screening tests, while this percentage was higher in the opt-out arm (3.6\%). ${ }^{46}$ The Australian national screening program already uses an approach where women are offered self-sampling if they decline an in-office visit. ${ }^{188}$

In low-resource settings, task shifting of cancer screening to community-based models could improve screening coverage and offer substantial cost savings through early detection and decreased labor costs, as we have already outlined in previous sections. In these settings, integration of cervical cancer screening with HIV monitoring ${ }^{189,190}$ could allow for greater cost-effectiveness in preventing both HPV and HIV infection treatment-related costs.

\section{Challenges and Limitations}

Introduction of a new screening practice or change in an existing screening program should follow the screening principles set by Wilson and Jungner in 1968 and consolidated lately by Dobrow et al. ${ }^{191,192}$ These principles offer guidance to implementation success by supporting the translation of evidence into practice in such a way that there is an optimal balance between benefits and harms of the new intervention. Consolidated principles are categorized into disease/condition, test/intervention, and program/ system principles. ${ }^{191}$ The main challenges and limitations of HPV self-sampling implementation as a test/intervention category are women's acceptance of self-sampling and HPV-test results, ensuring the use of only validated 
devices/assays, and ensuring adequate follow-up for all screen-positive women, expanded on as follows.

Acceptance Despite the fact that self-sampling and HPV testing is widely accepted by women of different ages, socioeconomic, cultural, and geographic backgrounds, barriers persist. In some settings, spousal consent is needed for screening and stigmatization in cases of HPV infection or cervical cancer and limits participation. Furthermore, women express concerns regarding test accuracy and whether they are performing the procedure correctly that need to be addressed to increase the acceptance of self-testing and receiving HPV-test results. Research should be focusing on developing culturally appropriate messages and educational materials aimed at both women and their spouses to address these challenges.

Validation of self-sampling devices/assays and standard operating procedures Studies evaluating the impact that individual self-sampling devices or components of the test kit (eg, instruction materials, in-person training, supervision) have on participation and acceptability are lacking. ${ }^{44}$ Clinical accuracy in the detection of highgrade cervical lesions using various commercially available self-sampling devices and PCR-based HPV assays needs to be systematically evaluated prior to implementation, since it is the most relevant criterion from a population-health standpoint. As more commercial HPV assays become available, validation and laboratory standard operating procedures assuring optimal sensitivity, specificity, and high intra- and interlaboratory reproducibility using international consensus criteria ${ }^{12}$ are needed for all assays intended for primary cervical cancer screening. $^{43,193}$

Course of action for screen-positive women Reflex triage of HPV-positive self-samples to cytologic examination could lower the burden of overtreatment and preterm births due to the shortening of the cervix after treatment. It could also lower the burden of screening on health-care workers and screening facilities. However, due to the increased complexity and cost of screening strategies with reflex triage and the need for follow-up of screennegative, triage-positive women, more research is needed on how different triage protocols perform in different local contexts, eg, HPV self-sampling screening with reflex triage and VIAC/treat approach for screen-positive and triage-positive women in low-resource settings. Clear and feasible guidelines should be in place on how to manage screen-positive/negative and triage-positive/negative women to assure their compliance and not to overburden health facilities.

Furthermore, the main challenges and limitations of HPV self-sampling implementation related to program/ system category that should guide further research in the field of implementation success of HPV self-sampling are as follows.

Information and participation More research is needed to understand the determinants of participation of different self-sampling implementation approaches and how to remove barriers to participation. Considering the pandemic's impact on the global economy, cost-effective, minimal-waste approaches should be investigated further. More trials are warranted to explore the effectiveness of the opt-in approach and potential avenues for improvement. Some of these improvement strategies may be campaigns to raise awareness of opting in to cervical cancer screening, offering electronic order options via text message, email, or mobile applications, and encouraging women to choose between self-sampling at home or clinician sampling. ${ }^{46}$ In some settings, an integrated approach could be beneficial, such as integrating cervical screening with women's monthly HIV therapy or cervical cancer screening of mothers with HPV vaccination of their children; however, more research is needed on the added value and feasibility of integrated guidelines, implementation and governance.

Continuity of care To ensure that improved screening coverage leads to improved health of screened women, it is necessary to enable rapid and free access to further highquality diagnostics, follow-up, and treatment for all women who participate in screening and are in need of those services. ${ }^{62}$ However, attendance at follow-up care after an HPV-positive self-sampling result has varied widely in high- and low-resource settings. To prevent loss to follow-up in women with a positive screening test, it is important to address the barriers women face in the local context, such as costs of care, lack of health insurance, distance to travel, time consumption, lack of health-care providers, and infrastructure. A communitybased approach and one-step (eg, screen and treat) approaches might add value in low-resource settings; however, only high-quality and evidence-based services should be implemented to minimize the treatment of women with false-positive and reassurance of women with false-negative results.

Overdiagnosis and overtreatment One of the concerns is related to the overdetection of cervical dysplasia 
related to high sensitivity of HPV testing as a screening strategy, resulting in increased overdiagnosing and overtreatment of transient infections. ${ }^{194}$ In low-resource settings with shortages of clinical facilities for follow-up and treatment and a shortage of adequately trained personnel, ${ }^{195}$ this is indeed worth noting. On the other hand, in most of these regions, the currently widely used low-cost visual assessment screening methods (such as VIAC) are already associated with overtreatment, due to the low specificity of these methods. ${ }^{196,197}$ It is also important to caution against alternative screening in young, unvaccinated women due to high HPV prevalence, which can be $20 \%-30 \%$ in women aged $<25$ years. In this population, an HPV-positive result offers a low positive predictive value. ${ }^{198}$ Marketing of these tests to young women in an opportunistic setting poses a challenge requiring adequate health-care professional follow-up and education for women to prevent overdiagnosis and overtreatment.

Infrastructure Another important concern when implementing HPV testing on self-samples in low-resource settings is the challenge of acquiring the necessary facilities and trained laboratory technicians for widespread HPV testing and appropriate quality control. One of the main barriers to the integration of highly accurate and robust HPV self-sampling into cervical cancer-screening programs in low-resource settings is the availability of highquality laboratory services. At the time that the WHO launched a global initiative to accelerate the elimination of cervical cancer in the midst of the pandemic on November 17, 2020, it was already clear that COVID-19 was not only a threat but also one of the most powerful opportunities we ever had to scale up HPV-based cervical cancer screening worldwide, due to the expansion of both the infrastructure and highly trained personnel currently working on COVID testing that could in time be allocated to increased HPV molecular testing capacity. With HPV self-sampling, more women could be reached through the expanded capacity of molecular laboratories across the world to accelerate the progress toward elimination of cervical cancer.

Economic evaluation of a comprehensive approach Important determinants of self-sampling cost-effectiveness are related to implementation approach (economic and ecological benefits of an opt-in approach versus higher participation in opt-out and community-outreach approaches), screening policy (age of women, screening interval), and guidelines for management of screen-positive women (triage, further diagnostics, follow-up, and treatment). Despite self-sampling in general being accepted as cost-effective, economic evaluation of selfsampling implementation should be performed in a local context, based on local screening strategy, its integration into a local health system, and local data (such as women's response to intervention, acceptability, availability of highquality care for women with a positive screening test results, and costs).

One size does not fit all Due to cultural differences across the world that are reflected in differences in acceptance of different screening approaches and technologies, differences in access to screening and high-quality care services in cases of positive screening results and the variability of the size effect of self-sampling trials in published studies, local trials have been recommended to assess feasibility, efficacy, and cost-effectiveness before progressing to a regional or national level. ${ }^{9}$

Governance COVID-19 has taught us that even in well-organized screening programs, a rapid and unexpected change in the environment can disrupt decadeslong, well-established dynamic equilibria of existing screening subsystems and stakeholder relations. Availability of data from dedicated screening-information systems and a clear governance structure is in such situations crucial for optimal adjustments to the program in order to minimize harms of screening disruptions and maintaining high-quality services when and where possible, as well as to plan recovery strategies. Having such structures in place is one of the prerequisites of implementation success, and should be considered and planned for in the preparatory phase.

\section{Conclusion}

Current evidence supports the use of HPV testing on selfsamples for overcoming barriers to screening and reaching underscreened women. Implementation of self-sampling needs to be comprehensive, appropriate for the local context, and cover everything from screening to treatment. Offering women a choice between different screening strategies can be beneficial, both in terms of increasing participation and cost-effectiveness. In low-resource settings, offering community-based self-sampling with educated personnel to assist with the process should be considered.

The COVID-19 pandemic represents an opportunity to accelerate progress toward elimination of cervical cancer if strategies are implemented with consideration of appropriate incorporation of diagnostics, follow-up, and treatment adapted to the setting. Infrastructure, personnel, and funds 
currently used for large-scale COVID-19 testing could in time be allocated to address disparities in cervical cancer burden and bring sensitive and robust cervical cancerscreening methods to resource-limited communities. Ensuring resilience of cervical cancer-screening efforts and health equity in access to screening should be one of the primary goals of designing and maintaining successful cervical cancer screening and prevention strategies in both highand low-income settings, as well as local communities.

\section{Author Contributions}

All authors have read and approved the final manuscript. All authors made a significant contribution to the work reported, whether in its conception, design, execution, acquisition of data, analysis and interpretation, or all these areas, took part in drafting, revising, or critically reviewing the article, gave final approval to the version to be published, have agreed on the journal to which the article has been submitted, and agree to be accountable for all aspects of the work.

\section{Funding}

This research received no external funding.

\section{Disclosure}

Megan Fitzpatrick is a scientific consultant for Digi8, which aims to optimize women's health, but has no direct relationship to the content presented herein. Dr. Fitzpatrick has received free reagents from Cepheid GeneXpert and Hologic ThinPrep vials for a prior study. The authors report no other conflicts of interest in this work.

\section{References}

1. Arbyn M, Weiderpass E, Bruni L, et al. Estimates of incidence and mortality of cervical cancer in 2018: a worldwide analysis. Lancet Glob Health. 2020;8:e191-e203. doi:10.1016/S2214-109X(19)304826

2. Arbyn M, Raifu AO, Weiderpass E, Bray F, Anttila A. Trends of cervical cancer mortality in the member states of the European Union. Eur $J$ Cancer. 2009;45:2640-2648. doi:10.1016/j. ejca.2009.07.018

3. Andrae B, Andersson TML, Lambert PC, et al. Screening and cervical cancer cure: population based cohort study. BMJ. 2012;344:e900e900. doi:10.1136/bmj.e900

4. Bos AB, Rebolj M, Habbema JDF, Van Ballegooijen M. Nonattendance is still the main limitation for the effectiveness of screening for cervical cancer in the Netherlands. Int $J$ Cancer. 2006;119:2372-2375. doi:10.1002/ijc.22114

5. Andrae B, Kemetli L, Sparén P, et al. Screening-preventable cervical cancer risks: evidence from a nationwide audit in Sweden. $J$ Natl Cancer Inst. 2008;100:622-629. doi:10.1093/jnci/djn099
6. Spence AR, Goggin P, Franco EL. Process of care failures in invasive cervical cancer: systematic review and meta-analysis. Prev Med (Baltim). 2007;45:93-106. doi:10.1016/j. ypmed.2007.06.007

7. Stenkvist B, Söderström J. Reasons for cervical cancer despite extensive screening. J Med Screen. 1996;3:204-207. doi:10.1177/ 096914139600300409

8. Ferlay J, Soerjomataram I, Dikshit R, et al. Cancer incidence and mortality worldwide: sources, methods and major patterns in GLOBOCAN 2012. Int $J$ Cancer. 2015;136:E359-E386. doi:10.1002/ijc. 29210

9. Arbyn M, Smith SB, Temin S, Sultana F, Castle P. Detecting cervical precancer and reaching underscreened women by using HPV testing on self samples: updated meta-analyses. BMJ. 2018; k4823. doi:10.1136/bmj.k4823

10. Verdoodt F, Jentschke M, Hillemanns P, Racey CS, Snijders PJF, Arbyn M. Reaching women who do not participate in the regular cervical cancer screening programme by offering self-sampling kits: a systematic review and meta-analysis of randomised trials. Eur J Cancer. 2015;51:2375-2385. doi:10.1016/j.ejca.2015.07.006

11. Hawkes D, Keung MHT, Huang Y, et al. Self-collection for cervical screening programs: from research to reality. Cancers (Basel). 2020;12:1053. doi:10.3390/cancers12041053

12. Meijer CJLM, Berkhof J, Castle PE, et al. Guidelines for human papillomavirus DNA test requirements for primary cervical cancer screening in women 30 years and older. Int $J$ Cancer. 2009;124:516-520. doi:10.1002/ijc. 24010

13. Arbyn M, Verdoodt F, Snijders PJF, et al. Accuracy of human papillomavirus testing on self-collected versus clinician-collected samples: a meta-analysis. Lancet Oncol. 2014;15:172-183. doi:10.1016/S1470-2045(13)70570-9

14. Vaccarella S, Lortet-Tieulent J, Plummer M, Franceschi S, Bray F. Worldwide trends in cervical cancer incidence: impact of screening against changes in disease risk factors. Eur J Cancer. 2013;49:3262-3273. doi:10.1016/j.ejca.2013.04.024

15. IARC. IARC Handbooks of Cancer Prevention Cervix Cancer Screening. IARC; 2005.

16. Arbyn M, Bruni L, Kelly D, et al. Tackling cervical cancer in Europe amidst the COVID-19 pandemic. Lancet Public Health. 2020;5:e425. doi:10.1016/S2468-2667(20)30122-5

17. Poljak M, Cuschieri K, Waheed DEN, Baay M, Vorsters A. Impact of the covid-19 pandemic on human papillomavirusbased testing services to support cervical cancer screening. Acta Dermatovenerologica Alpina Pannonica Adriat. 2021;30:21-26. doi:10.15570/actaapa.2021.5.

18. Arbyn M, Gultekin M, Morice P, et al. The European response to the WHO call to eliminate cervical cancer as a public health problem. Int J Cancer. 2021;148:277-284. doi:10.1002/ijc.33189

19. WHO NCD Department. Final Results Rapid Assessment of Service Delivery for Noncommunicable Disease During the COVID-19 Pandemic. WHO; 2020.

20. Epic Health Research Network. Delayed cancer screeningsa second look; 2021. https://ehrn.org/articles/delayed-cancerscreenings-a-second-look//index.html. Accessed May 1, 2021.

21. Ivanuš $U$, Jerman $T$, Gašper Oblak $U$, et al. The impact of the COVID-19 pandemic on organised cervical cancer screening: the first results of the Slovenian cervical screening programme and registry. Lancet Reg Health Eur. 2021;5:100101. doi:10.1016/j. lanepe.2021.100101

22. Australian Institute of Health and Welfare. Cancer screening and COVID-19 in Australia. 2021

23. Dinmohamed AG, Visser O, Verhoeven RHA, et al. Fewer cancer diagnoses during the COVID-19 epidemic in the Netherlands. Lancet Oncol. 2020;21:750-751. doi:10.1016/S1470-2045(20) 30265-5 
24. Tsibulak I, Reiser E, Bogner G, et al. Decrease in gynecological cancer diagnoses during the COVID-19 pandemic: an Austrian perspective. Int $J$ Gynecol Cancer. 2020;30:1667-1671. doi:10.1136/ijgc-2020-001975

25. Skovlund CW, Friis S, Dehlendorff C, Nilbert MC, Mørch LS. Hidden morbidities: drop in cancer diagnoses during the COVID-19 pandemic in Denmark. Acta Oncol (Madr). 2021;60:20-23. doi:10.1080/0284186X.2020.1858235

26. Jacob L, Loosen SH, Kalder M, Luedde T, Roderburg C, Kostev K. Impact of the COVID-19 pandemic on cancer diagnoses in general and specialized practices in Germany. Cancers (Basel). 2021;13:408. doi:10.3390/cancers13030408

27. Maluchnik M, Podwójcic K, Więckowska B. Decreasing access to cancer diagnosis and treatment during the COVID-19 pandemic in Poland. Acta Oncol (Madr). 2021;60:28-31. doi:10.1080/ 0284186X.2020.1837392

28. Castanon A, Rebolj M, Pesola F, Sasieni P. Recovery strategies following COVID-19 disruption to cervical cancer screening and their impact on excess diagnoses. $\mathrm{Br} J$ Cancer. 2021;124:1361-1365. doi:10.1038/s41416-021-01275-3

29. Miller MJ, Xu L, Qin J, et al. Impact of COVID-19 on cervical cancer screening rates among women aged 21-65 years in a large integrated health care system - Southern California, January 1September 30, 2019, and January 1-September 30, 2020. MMWR Morb Mortal Wkly Rep. 2021;70:109-113. doi:10.15585/mmwr. mm7004a1

30. Uppal S, Chapman C, Spencer RJ, et al. Association of hospital volume with racial and ethnic disparities in locally advanced cervical cancer treatment. Obstet Gynecol. 2017;129:295-304. doi:10.1097/AOG.0000000000001819

31. White A, Thompson TD, White MC, et al. Cancer screening test use - United States, 2015. MMWR Morb Mortal Wkly Rep. 2017;66:201-206. doi:10.15585/mmwr.mm6608a1

32. Beavis AL, Gravitt PE, Rositch AF. Hysterectomy-corrected cervical cancer mortality rates reveal a larger racial disparity in the United States. Cancer. 2017;123:1044-1050. doi:10.1002/ cncr.30507

33. Tracy JK, Lydecker AD, Ireland L. Barriers to cervical cancer screening among lesbians. $J$ Womens Health (Larchmt). 2010;19:229-237. doi:10.1089/jwh.2009.1393

34. Paskett ED, McLaughlin JM, Reiter PL, et al. Psychosocial predictors of adherence to risk-appropriate cervical cancer screening guidelines: a cross sectional study of women in Ohio Appalachia participating in the Community Awareness Resources and Education (CARE) project. Prev Med (Baltim). 2010;50:74-80. doi:10.1016/j.ypmed.2009.09.001

35. Cancino RS, Su Z, Mesa R, Tomlinson GE, Wang J. The impact of COVID-19 on cancer screening: challenges and opportunities. JMIR Cancer. 2020;6:e21697. doi:10.2196/21697

36. MacLaughlin KL, Jacobson RM, Radecki Breitkopf C, et al. Trends over time in pap and pap-HPV cotesting for cervical cancer screening. $J$ Womens Health. 2019;28:244-249. doi:10.1089/jwh.2018.7380

37. Thompson D, Lei Y. Mini review: recent progress in RT-LAMP enabled COVID-19 detection. Sensor Actuator Rep. 2020;2:100017. doi:10.1016/j.snr.2020.100017

38. Gao J, Wu L, Yang D, Gong W, Wang J, One-Pot A. CRISPR/ Cas9-typing PCR for DNA detection and genotyping. $J$ Mol Diagn. 2021;23:46-60. doi:10.1016/j.jmoldx.2020.10.004

39. Ramachandran A, Huyke DA, Sharma E, et al. Electric field-driven microfluidics for rapid CRISPR-based diagnostics and its application to detection of SARS-CoV-2. Proc Natl Acad Sci U S A. 2020;117:29518-29525. doi:10.1073/pnas.2010254117
40. Sigurdsson K, Hrafnkelsson J, Geirsson G, Gudmundsson J, Salvarsdóttir A. Screening as a prognostic factor in cervical cancer: analysis of survival and prognostic factors based on Icelandic population data, 1964-1988. Gynecol Oncol. 1991;43:64-70. doi:10.1016/0090-8258(91)90011-S

41. Landy R, Pesola F, Castañón A, Sasieni P. Impact of cervical screening on cervical cancer mortality: estimation using stage-specific results from a nested case-control study. $\mathrm{Br}$ $J$ Cancer. 2016;115:1140-1146. doi:10.1038/bjc.2016.290

42. Takač I, Uršič-Vrščaj M, Repše-Fokter A, et al. Clinicopathological characteristics of cervical cancer between 2003 and 2005, after the introduction of a national cancer screening program in Slovenia. Eur J Obstet Gynecol Reprod Biol. 2008;140:82-89. doi:10.1016/j.ejogrb.2008.02.019

43. Arbyn M, Castle PE. Offering self-sampling kits for HPV testing to reach women who do not attend in the regular cervical cancer screening program. Cancer Epidemiol Biomarkers Prev. 2015;24:769-772. doi:10.1158/1055-9965.EPI-14-1417

44. Yeh PT, Kennedy CE, De Vuyst H, Narasimhan M. Self-sampling for human papillomavirus (HPV) testing: a systematic review and meta-analysis. BMJ Glob Health. 2019;4:e001351. doi:10.1136/ bmjgh-2018-001351

45. Sarai racey C, Withrow DR, Gesink D. Self-collected HPV testing improves participation in cervical cancer screening: a systematic review and meta-analysis. Can J Public Health. 2013;104(2):e159-e166. doi:10.1007/bf03405681

46. Ivanus U, Jerman T, Fokter AR, et al. Randomised trial of HPV self-sampling among non-attenders in the Slovenian cervical screening programme ZORA: comparing three different screening approaches. Radiol Oncol. 2018;52:399-412. doi:10.2478/raon2018-0036

47. Broberg G, Gyrd-Hansen D, Miao Jonasson J, et al. Increasing participation in cervical cancer screening: offering a HPV self-test to long-term non-attendees as part of RACOMIP, a Swedish randomized controlled trial. Int $J$ Cancer. 2014;134:2223-2230. doi:10.1002/ijc. 28545

48. Tranberg M, Bech BH, Blaakær J, Jensen JS, Svanholm H, Andersen B. Preventing cervical cancer using HPV self-sampling: direct mailing of test-kits increases screening participation more than timely opt-in procedures - a randomized controlled trial. BMC Cancer. 2018;18:1. doi:10.1186/s12885-018-4165-4.

49. Chao YS. HPV self-sampling for primary cervical cancer screening: a review of diagnostic test accuracy and clinical evidence an update. Can Agency Drugs Technol Health. 2019.

50. Forte T, Lockwood GA, McLachlin CM, Fekete S, Bryant HE. A first look at participation rates in cervical cancer screening programs in Canada. Curr Oncol. 2012;19:269-271. doi:10.3747/co.19.1188

51. Bishop E, Katz ML, Reiter PL. Acceptability of human papillomavirus self-sampling among a national sample of women in the United States. Biores Open Access. 2019;8:65-73. doi:10.1089/biores.2018.0040

52. Madzima TR, Vahabi M, Lofters A. Emerging role of HPV self-sampling in cervical cancer screening for hard-To-reach women. Can Fam Physician. 2017;63(8):597-601.

53. Scarinci IC, Litton AG, Garcés-Palacio IC, Partridge EE, Castle PE. Acceptability and usability of self-collected sampling for HPV testing among African-American women living in the Mississippi delta. Womens Health Issues. 2013;23:e123-e130. doi:10.1016/j.whi.2012.12.003

54. Vanderpool RC, Jones MG, Stradtman LR, Smith JS, Crosby RA. Self-collecting a cervico-vaginal specimen for cervical cancer screening: an exploratory study of acceptability among medically underserved women in rural Appalachia. Gynecol Oncol. 2014;132:S21-S25. doi:10.1016/j.ygyno.2013.10.008 
55. Reiter P, Richardson M. Acceptability of human papillomavirus self-test devices among women from high-risk populations. $J$ Womens Health Issues Care. 2016;5(1). doi:10.4172/23259795.1000216

56. Richman AR, Brewer NT, Liebman AK, Rinas AC, Smith JS. Optimising human papillomavirus self-testing for high risk women. Sex Transm Infect. 2011;87:118-122. doi:10.1136/ sti.2010.046326

57. Katz ML, Zimmermann BJ, Moore D, Paskett ED, Reiter PL. Perspectives from health-care providers and women about completing human papillomavirus (HPV) self-testing at home. Women Health. 2017;57:1161-1177. doi:10.1080/03630242.20 16.1243608

58. Reiter PL, McRee AL. Cervical cancer screening (Pap testing) behaviours and acceptability of human papillomavirus self-testing among lesbian and bisexual women aged 21-26 years in the USA. J Fam Plan Reprod Heal Care. 2015;41:259-264. doi:10.1136/ jfprhc-2014-101004

59. Winer RL, Lin J, Tiro JA, et al. Effect of mailed human papillomavirus test kits vs usual care reminders on cervical cancer screening uptake, precancer detection, and treatment: a randomized clinical trial. JAMA Netw Open. 2019;2:e1914729. doi:10.1001/jamanetworkopen.2019.14729

60. Racey CS, Gesink DC, Burchell AN, Trivers S, Wong T, Rebbapragada A. Randomized intervention of self-collected sampling for human papillomavirus testing in under-screened rural women: uptake of screening and acceptability. $J$ Womens Health. 2016;25:489-497. doi:10.1089/jwh.2015.5348

61. Castle PE, Rausa A, Walls T, et al. Comparative community outreach to increase cervical cancer screening in the Mississippi Delta. Prev Med (Baltim). 2011;52:452-455. doi:10.1016/j. ypmed.2011.03.018

62. Organización Mundial de la Salud - OMS. WHO Guidelines for Screening and Treatment of Precancerous Lesions for Cervical Cancer Prevention. WHO; 2013.

63. Ginsburg O, Badwe R, Boyle P, et al. Changing global policy to deliver safe, equitable, and affordable care for women's cancers. Lancet. 2017;389:871-880. doi:10.1016/S0140-6736(16)31393-9

64. Kamath Mulki A, Withers M. Human papilloma virus self-sampling performance in low- and middle-income countries. BMC Womens Health. 2021;21:1. doi:10.1186/s12905-02001158-4.

65. Sancho-Garnier H, Tamalet C, Halfon P, et al. HPV self-sampling or the Pap-smear: a randomized study among cervical screening nonattenders from lower socioeconomic groups in France. Int J Cancer. 2013;133(11):2681-2687. doi:10.1002/ijc.28283

66. Cadman L, Wilkes S, Mansour D, et al. A randomized controlled trial in non-responders from Newcastle upon Tyne invited to return a self-sample for Human Papillomavirus testing versus repeat invitation for cervical screening. $J$ Med Screen. 2015;22:28-37. doi:10.1177/0969141314558785

67. Montealegre JR, Mullen PD, Jibaja-Weiss M, Vargas Mendez MM, Scheurer ME. Feasibility of cervical cancer screening utilizing self-sample human papillomavirus testing among Mexican immigrant women in Harris County, Texas: a pilot study. J Immigr Minor Health. 2015;17:704-712. doi:10.1007/ s10903-014-0125-5

68. Abuelo CE, Levinson KL, Salmeron J, Sologuren CV, Fernandez MJV, Belinson JL. The peru cervical cancer screening study (PERCAPS): the design and implementation of a mother/ daughter screen, treat, and vaccinate program in the peruvian jungle. J Community Health. 2014;39:409-415. doi:10.1007/ s10900-013-9786-6
69. Fitzpatrick MB, El-Khatib Z, Katzenstein D, Pinsky BA, Chirenje ZM, McCarty K. Community-based self-collected human papillomavirus screening in rural Zimbabwe. $B M C$ Public Health. 2019;19:1-8. doi:10.1186/s12889-019-6810-5.

70. Trope LA, Chumworathayi B, Blumenthal PD. Feasibility of community-based careHPV for cervical cancer prevention in rural Thailand. J Low Genit Tract Dis. 2013;17:315-319. doi:10.1097/LGT.0b013e31826b7b70

71. Nakalembe M, Makanga P, Kambugu A, Laker-Oketta M, Huchko MJ, Martin J. A public health approach to cervical cancer screening in Africa through community-based self-administered HPV testing and mobile treatment provision. Cancer Med. 2020;9:8701-8712. doi:10.1002/cam4.3468

72. Gök M, Van Kemenade FJ, Heideman DAM, et al. Experience with high-risk human papillomavirus testing on vaginal brush-based self-samples of non-attendees of the cervical screening program. Int J Cancer. 2012;130:1128-1135. doi:10.1002/ ijc. 26128

73. Bray F, Ferlay J, Soerjomataram I, Siegel RL, Torre LA, Jemal A. Global cancer statistics 2018: GLOBOCAN estimates of incidence and mortality worldwide for 36 cancers in 185 countries. CA Cancer J Clin. 2018;68(6):394-424. doi:10.3322/caac.21492

74. Gakidou E, Nordhagen S, Obermeyer Z. Coverage of cervical cancer screening in 57 countries: low average levels and large inequalities. PLoS Med. 2008;5:e132. doi:10.1371/journal. pmed.0050132

75. Sankaranarayanan R. Overview of cervical cancer in the developing world. Int $J$ Gynecol Obstet. 2006;95:S205-S210. doi:10.1016/S0020-7292(06)60035-0

76. Ndejjo R, Mukama T, Musabyimana A, Musoke D. Uptake of cervical cancer screening and associated factors among women in rural Uganda: a cross sectional study. PLoS One. 2016;11: e0149696. doi:10.1371/journal.pone.0149696

77. Kuguyo O, Matimba A, Tsikai N, et al. Cervical cancer in Zimbabwe: a situation analysis. Pan Afr Med J. 2017;27:215. doi:10.11604/pamj.2017.27.215.12994

78. Research WA for HP and S. Prevention of cervical cancer through screening using visual inspection with acetic acid (VIA) and treatment with cryotherapy. Outlook. 2003;II(1):33.

79. Bin LJ, Simms KT, Smith MA, et al. Primary HPV testing versus cytology-based cervical screening in women in Australia vaccinated for HPV and unvaccinated: effectiveness and economic assessment for the National Cervical Screening Program. Lancet Public Health. 2017;2(2):e96-e107. doi:10.1016/S2468-2667(17) 30007-5

80. Tapera O, Kadzatsa W, Nyakabau AM, et al. Sociodemographic inequities in cervical cancer screening, treatment and care amongst women aged at least 25 years: evidence from surveys in Harare, Zimbabwe. BMC Public Health. 2019;19:1-2. doi:10.1186/s12889-019-6749-6

81. Nabirye J, Okwi LA, Nuwematsiko R, et al. Health system factors influencing uptake of Human Papilloma Virus (HPV) vaccine among adolescent girls 9-15 years in Mbale District, Uganda. BMC Public Health. 2020;20:1. doi:10.1186/s12889-020-8302-z

82. Allen-Leigh $\mathrm{B}$, Uribe-Zúñiga $\mathrm{P}$, León-Maldonado $\mathrm{L}$, et al. Barriers to HPV self-sampling and cytology among low-income indigenous women in rural areas of a middle-income setting: a qualitative study. BMC Cancer. 2017;17:1. doi:10.1186/ s12885-017-3723-5

83. Brandt T, Wubneh SB, Handebo S, et al. Genital self-sampling for HPV-based cervical cancer screening: a qualitative study of preferences and barriers in rural Ethiopia. BMC Public Health. 2019;19:1-9. doi:10.1186/s12889-019-7354-4 
84. Campos NG, Mvundura M, Jeronimo J, Holme F, Vodicka E, Kim JJ. Cost-effectiveness of HPV-based cervical cancer screening in the public health system in Nicaragua. BMJ Open. 2017;7: e015048. doi:10.1136/bmjopen-2016-015048

85. Termrungruanglert W, Khemapech N, Tantitamit T, Sangrajrang S, Havanond P, Laowahutanont P. Cost-effectiveness analysis study of HPV testing as a primary cervical cancer screening in Thailand. Gynecol Oncol Rep. 2017;22:58-63. doi:10.1016/j.gore.2017.09.007

86. Awua AK, Wiredu EK, Afari EA, Tijani AS, Djanmah G, Adanu RMK. A tailored within-community specimen collection strategy increased uptake of cervical cancer screening in a cross-sectional study in Ghana. BMC Public Health. 2017;18:1. doi:10.1186/s12889-017-4631-y

87. Huchko MJ, Ibrahim S, Blat C, et al. Cervical cancer screening through human papillomavirus testing in community health campaigns versus health facilities in rural western Kenya. Int J Gynecol Obstet. 2018;141:63-69. doi:10.1002/ijgo.12415

88. Fitzpatrick MB, Dube Mandishora RS, Katzenstein DA, et al. hrHPV prevalence and type distribution in rural Zimbabwe: a community-based self-collection study using near-point-of-care GeneXpert HPV testing. Int J Infect Dis. 2019;82:21-29. doi:10.1016/j.ijid.2019.02.022

89. Fitzpatrick M, Pathipati MP, McCarty K, et al. Knowledge, attitudes, and practices of cervical Cancer screening among HIV-positive and HIV-negative women participating in human papillomavirus screening in rural Zimbabwe. BMC Womens Health. 2020;20:1. doi:10.1186/s12905-020-01017-2

90. Bansil P, Wittet S, Lim JL, Winkler JL, Paul P, Jeronimo J. Acceptability of self-collection sampling for HPV-DNA testing in low-resource settings: a mixed methods approach. BMC Public Health. 2014;14:1-9. doi:10.1186/1471-2458-14-596.

91. Arriba LN, Enerson CL, Belinson S, Novick L, Belinson J. Mexican cervical cancer screening study ii: acceptability of human papillomavirus self-sampler. Int $J$ Gynecol Cancer. 2010;20(8):1415-23. doi:10.1111/IGC.0b013e3181f58678

92. Arbyn M, Depuydt C, Benoy I, et al. VALGENT: a protocol for clinical validation of human papillomavirus assays. J Clin Virol. 2016;76:S14-S21. doi:10.1016/j.jcv.2015.09.014

93. Dutch National Institute for Public Health and the Environment. Annual Report RIVM. 2020.

94. Smith M, Bin LJ, Simms K, Canfell K. Impact of HPV sample self-collection for underscreened women in the renewed cervical screening program. Med J Aust. 2016;204:194. doi:10.5694/ mja15.00912

95. von Karsa L, Arbyn M, De VH, et al. European guidelines for quality assurance in cervical cancer screening - second edition supplements. Papillomavir Res. 2015;1:22-31. doi:10.1016/j. pvr.2015.06.006

96. Karjalainen L, Anttila A, Nieminen P, Luostarinen T, Virtanen A. Self-sampling in cervical cancer screening: comparison of a brush-based and a lavage-based cervicovaginal self-sampling device. BMC Cancer. 2016;16:1. doi:10.1186/ s12885-016-2246-9.

97. Virtanen A, Anttila A, Luostarinen T, Nieminen P. Self-sampling versus reminder letter: effects on cervical cancer screening attendance and coverage in Finland. Int $J$ Cancer. 2011;128:2681-2687. doi:10.1002/ijc.25581

98. Polman NJ, Ebisch RMF, Heideman DAM, et al. Performance of human papillomavirus testing on self-collected versus clinician-collected samples for the detection of cervical intraepithelial neoplasia of grade 2 or worse: a randomised, paired screen-positive, non-inferiority trial. Lancet Oncol. 2019;20:229-238. doi:10.1016/S1470-2045(18)30763-0
99. Smith MA, Hall MT, Saville M, et al. Could HPV testing on self-collected samples be routinely used in an organized cervical screening program? A modeled analysis. Cancer Epidemiol Biomarkers Prev. 2021;30:268-277. doi:10.1158/1055-9965.EPI20-0998

100. Bais AG, Van Kemenade FJ, Berkhof J, et al. Human papillomavirus testing on self-sampled cervicovaginal brushes: an effective alternative to protect nonresponders in cervical screening programs. Int $J$ Cancer. 2007;120:1505-1510. doi:10.1002/ ijc. 22484

101. Lazcano-Ponce E, Lorincz AT, Cruz-Valdez A, et al. Self-collection of vaginal specimens for human papillomavirus testing in cervical cancer prevention (MARCH): a community-based randomised controlled trial. Lancet. 2011;378:1868-1873. doi:10.1016/ S0140-6736(11)61522-5

102. Bosgraaf RP, Verhoef VMJ, Massuger LFAG, et al. Comparative performance of novel self-sampling methods in detecting high-risk human papillomavirus in 30,130 women not attending cervical screening. Int $J$ Cancer. 2015;136(3):646-655. doi:10.1002/ijc. 29026

103. Szarewski A, Cadman L, Mesher D, et al. HPV self-sampling as an alternative strategy in non-attenders for cervical screeninga randomised controlled trial. Br J Cancer. 2011;104:915-920. doi:10.1038/bjc. 2011.48

104. Wikström I, Lindell M, Sanner K, Wilander E. Self-sampling and HPV testing or ordinary Pap-smear in women not regularly attending screening: a randomised study. $\mathrm{Br} J$ Cancer. 2011;105:337-339. doi:10.1038/bjc.2011.236

105. Surriabre P, Allende G, Prado M, et al. Self-sampling for human papillomavirus DNA detection: a preliminary study of compliance and feasibility in BOLIVIA. BMC Womens Health. 2017;17:1-7. doi:10.1186/s12905-017-0490-z

106. Gök M, Heideman DAM, Van Kemenade FJ, et al. HPV testing on self collected cervicovaginal lavage specimens as screening method for women who do not attend cervical screening: cohort study. BMJ. 2010;340:c1040-c1040. doi:10.1136/bmj.c1040

107. Giorgi Rossi P, Marsili LM, Camilloni L, et al. The effect of self-sampled HPV testing on participation to cervical cancer screening in Italy: a randomised controlled trial (ISRCTN96071600). $\mathrm{Br} \quad J \quad$ Cancer. 2011;104:248-254. doi: $10.1038 /$ sj.bjc. 6606040

108. Virtanen A, Nieminen P, Luostarinen T, Anttila A. Self-sample HPV tests as an intervention for nonattendees of cervical cancer screening in Finland: a randomized trial. Cancer Epidemiol Biomarkers Prev. 2011;20:1960-1969. doi:10.1158/1055-9965.EPI-11-0307

109. Ogilvie GS, Patrick DM, Schulzer M, et al. Diagnostic accuracy of self collected vaginal specimens for human papillomavirus compared to clinician collected human papillomavirus specimens: a meta-analysis. Sex Transm Infect. 2005;81:207-212. doi:10.1136/sti.2004.011858

110. Hillemanns P, Kimmig R, Hüttemann U, Dannecker C, Thaler CJ. Screening for cervical neoplasia by self-assessment for human papillomavirus DNA. Lancet. 1999;354:1970. doi:10.1016/ S0140-6736(99)04110-0

111. Dannecker C, Siebert U, Thaler CJ, Kiermeir D, Hepp H, Hillemanns P. Primary cervical cancer screening by self-sampling of human papillomavirus DNA in internal medicine outpatient clinics. Ann Oncol. 2004;15:863-869. doi:10.1093/ annonc/mdh 240

112. Sellors JW, Lorincz AT, Mahony JB, et al. Comparison of self-collected vaginal, vulvar and urine samples with physician-collected cervical samples for human papillomavirus testing to detect high-grade squamous intraepithelial lesions. CMAJ. 2000;163(5):513-518. 
113. Szarewski A, Cadman L, Ashdown-Barr L, Waller J. Exploring the acceptability of two self-sampling devices for human papillomavirus testing in the cervical screening context: a qualitative study of Muslim women in London. $J$ Med Screen. 2009;16:193-198. doi:10.1258/jms.2009.009069

114. Leinonen MK, Schee K, Jonassen CM, et al. Safety and acceptability of human papillomavirus testing of self-collected specimens: a methodologic study of the impact of collection devices and HPV assays on sensitivity for cervical cancer and high-grade lesions. J Clin Virol. 2018;99-100:22-30. doi:10.1016/j.jcv.2017.12.008

115. Enerly E, Bonde J, Schee K, Pedersen H, Lönnberg S, Nygård M. Self-sampling for human papillomavirus testing among non-attenders increases attendance to the Norwegian cervical cancer screening programme. PLoS One. 2016;11:e151978. doi:10.1371/journal.pone. 0151978

116. Lefeuvre C, Pivert A, Guillou-Guillemette H, et al. Urinary HPV DNA testing as a tool for cervical cancer screening in women who are reluctant to have a Pap smear in France. $J$ Infect. 2020;81:248-254. doi:10.1016/j.jinf.2020.05.006

117. Van Keer S, Tjalma WAA, Pattyn J, et al. Human papillomavirus genotype and viral load agreement between paired first-void urine and clinician-collected cervical samples. Eur J Clin Microbiol Infect Dis. 2018;37:859-869. doi:10.1007/s10096-017-3179-1

118. Sargent A, Fletcher S, Bray K, Kitchener HC, Crosbie EJ. Crosssectional study of HPV testing in self-sampled urine and comparison with matched vaginal and cervical samples in women attending colposcopy for the management of abnormal cervical screening. BMJ Open. 2019;9:e025388. doi:10.1136/bmjopen-2018-025388

119. Cadman L, Reuter C, Jitlal M, et al. A randomized comparison of different vaginal self-sampling devices and urine for human papillomavirus testing-predictors 5.1. Cancer Epidemiol Biomarkers Prev. 2021;30:661-668. doi:10.1158/1055-9965.EPI-20-1226

120. Tranberg M, Jensen JS, Bech BH, Andersen B. Urine collection in cervical cancer screening - analytical comparison of two HPV DNA assays. BMC Infect Dis. 2020;20:1. doi:10.1186/s12879-020-05663-7.

121. Pathak N, Dodds J, Zamora J, Khan K. Accuracy of urinary human papillomavirus testing for presence of cervical HPV: systematic review and meta-analysis. BMJ. 2014;349:g5264-g5264. doi:10.1136/bmj.g5264

122. Ørnskov D, Jochumsen K, Steiner PH, Grunnet IM, Lykkebo AW, Waldstrøm M. Clinical performance and acceptability of self-collected vaginal and urine samples compared with clinician-taken cervical samples for hpv testing among women referred for colposcopy. a cross-sectional study. BMJ Open. 2021;11:e041512. doi:10.1136/bmjopen-2020-041512

123. Stanczuk GA, Currie H, Baxter G, et al. Cobas 4800 HPV detection in the cervical, vaginal and urine samples of women with high-grade CIN before and after treatment. $J$ Clin Pathol. 2015;68:567-570. doi:10.1136/jclinpath-2014-202851

124. Cho HW, Ouh YT, Hong JH, et al. Comparison of urine, self-collected vaginal swab, and cervical swab samples for detecting human papillomavirus (HPV) with Roche Cobas HPV, Anyplex II HPV, and RealTime HR-S HPV assay. $J$ Virol Methods. 2019;269:77-82. doi:10.1016/j.jviromet.2019.04.012

125. Cuzick J, Cadman L, Ahmad AS, et al. Performance and diagnostic accuracy of a urine-based human papillomavirus assay in a referral population. Cancer Epidemiol Biomarkers Prev. 2017;26:1053-1059. doi:10.1158/1055-9965.EPI-16-0960

126. Colli-Pee: An innovative solution for non-invasive urine collection and stabilization [homepage on the Internet]. Novosanis. Available from: https://novosanis.com/our-products. Accessed May 16, 2021.

127. Huynh J, Howard M, Lytwyn A. Self-collection for vaginal human papillomavirus testing: systematic review of studies asking women their perceptions. $J$ Low Genit Tract Dis. 2010;14:356-362. doi:10.1097/LGT.0b013e3181dc115b
128. Igidbashian S, Boveri S, Spolti N, Radice D, Sandri MT, Sideri M. Self-collected human papillomavirus testing acceptability: comparison of two self-sampling modalities. $J$ Womens Health. 2011;20:397-402. doi:10.1089/jwh.2010.2189

129. Wikström I, Stenvall H, Wilander E. Attitudes to self-sampling of vaginal smear for human papilloma virus analysis among women not attending organized cytological screening. Acta Obstet Gynecol Scand. 2007;86:720-725. doi:10.1080/0001634070 1303747

130. Polman NJ, de Haan Y, Veldhuijzen NJ, et al. Experience with HPV self-sampling and clinician-based sampling in women attending routine cervical screening in the Netherlands. Prev Med (Baltim). 2019;125:5-11. doi:10.1016/j.ypmed.2019.04.025

131. Shin HY, Lee B, Hwang SH, et al. Evaluation of satisfaction with three different cervical cancer screening modalities: clinician-collected pap test vs. HPV test by self-sampling vs. HPV test by urine sampling. J Gynecol Oncol. 2019;30(5):e76. doi:10.3802/jgo.2019.30.e76

132. Sultana F, Mullins R, Murphy M, et al. Women's views on human papillomavirus self-sampling: focus groups to assess acceptability, invitation letters and a test kit in the Australian setting. Sex Health. 2015;12:279. doi:10.1071/SH14236

133. Ketelaars PJW, Bosgraaf RP, Siebers AG, et al. High-risk human papillomavirus detection in self-sampling compared to physician-taken smear in a responder population of the Dutch cervical screening: results of the VERA study. Prev Med (Baltim). 2017;101:96-101. doi:10.1016/j.ypmed.2017.05.021

134. Virtanen A, Nieminen P, Niironen M, Luostarinen T, Anttila A. Self-sampling experiences among non-attendees to cervical screening. Gynecol Oncol. 2014;135:487-494. doi:10.1016/j. ygyno.2014.09.019

135. Arrossi S, Ramos S, Straw C, Thouyaret L, Orellana L. HPV testing: a mixed-method approach to understand why women prefer self-collection in a middle-income country. BMC Public Health. 2016;16:1. doi:10.1186/s12889-016-3474-2.

136. Waller J, McCaffery K, Forrest S, et al. Acceptability of unsupervised HPV self-sampling using written instructions. $J$ Med Screen. 2006;13:208-213. doi:10.1177/096914130601300409

137. Castle PE, Silva VRS, Consolaro MEL, et al. Participation in cervical screening by selfcollection, pap, or a choice of either in Brazil. Cancer Prev Res. 2019;12:159-170. doi:10.1158/19406207.CAPR-18-0419

138. Kellen E, Benoy I, Vanden Broeck D, et al. A randomized, controlled trial of two strategies of offering the home-based HPV self-sampling test to non- participants in the Flemish cervical cancer screening program. Int $J$ Cancer. 2018;143:861-868. doi:10.1002/ijc.31391

139. Montealegre JR, Landgren RM, Anderson ML, et al. Acceptability of self-sample human papillomavirus testing among medically underserved women visiting the emergency department. Gynecol Oncol. 2015;138:317-322. doi:10.1016/j. ygyno.2015.05.028

140. Sultana F, Mullins R, English DR, et al. Women's experience with home-based self-sampling for human papillomavirus testing. BMC Cancer. 2015;15:1. doi:10.1186/s12885-015-1804-x

141. Bosgraaf RP, Ketelaars PJW, Verhoef VMJ, et al. Reasons for non-attendance to cervical screening and preferences for HPV self-sampling in Dutch women. Prev Med (Baltim). 2014;64:108-113. doi:10.1016/j.ypmed.2014.04.011

142. Forrest S, McCaffery K, Waller J, et al. Attitudes to self-sampling for HPV among Indian, Pakistani, African-Caribbean and white British women in Manchester, UK. J Med Screen. 2004;11:85-88. doi:10.1258/096914104774061065

143. Kahn JA, Bernstein DI, Rosenthal SL, et al. Acceptability of human papillomavirus self testing in female adolescents. Sex Transm Infect. 2005;81:408-414. doi:10.1136/sti.2004.012047 
144. Crofts V, Flahault E, Tebeu PM, et al. Education efforts may contribute to wider acceptance of human papillomavirus self-sampling. Int J Womens Health. 2015;7:149. doi:10.2147/ IJWH.S56307.

145. Chen SL, Hsieh PC, Chou CH, Tzeng YL. Determinants of women's likelihood of vaginal self-sampling for human papillomavirus to screen for cervical cancer in Taiwan: a cross-sectional study. BMC Womens Health. 2014;14:1-7. doi:10.1186/s12905014-0139-0

146. Galbraith KV, Gilkey MB, Smith JS, Richman AR, Barclay L, Brewer NT. Perceptions of mailed HPV self-testing among women at higher risk for cervical cancer. $J$ Community Health. 2014;39:849-856. doi:10.1007/s10900-014-9931-x

147. Ndukwe EG, Williams KP, Sheppard V. Knowledge and perspectives of breast and cervical cancer screening among female African immigrants in the Washington D.C. metropolitan area. J Cancer Educ. 2013;28:748-754. doi:10.1007/s13187-013-0521-x

148. Frota da Rocha Morgado F, Kopp Xavier da Silveira EM, Pinheiro Rodrigues Do Nascimento L, et al. Psychometric assessment of the EMIC Stigma Scale for Brazilians affected by leprosy. PLoS One. 2020;15:e0239186. doi:10.1371/journal. pone.0239186

149. Were E, Nyaberi Z, Buziba N. Perceptions of risk and barriers to cervical cancer screening at Moi Teaching and Referral Hospital (MTRH), Eldoret, Kenya. Afr Health Sci. 2011;11(1):58-64.

150. Fokom Defo V, Fokom Domgue J. Why consider self-sampling for cervical cancer screening in low- and middle-income countries? JAMA Ethics. 2020;22(2):116-125. doi:10.1001/ amajethics.2020.116

151. Campos NG, Alfaro K, Maza M, et al. The cost-effectiveness of human papillomavirus self-collection among cervical cancer screening non-attenders in El Salvador. Prev Med (Baltim). 2020;131:105931. doi:10.1016/j.ypmed.2019.105931

152. Ho IK, Dinh KT. Cervical cancer screening among Southeast Asian American Women. J Immigr Minor Health. 2011;13 (1):49-60. doi:10.1007/s10903-010-9358-0

153. Mangold BR. Self-collected samples in cervical cancer screening: results of HPV and pap self-collected samples compared to physician-obtained specimens. Acta Cytol. 2019;63:379-384. doi:10.1159/000499373

154. Garcia F, Barker B, Santos C, et al. Cross-sectional study of patient- and physician-collected cervical cytology and human papillomavirus. Obstet Gynecol. 2003;102(2):266-272. doi:10.1016/S0029-7844(03)00517-9

155. Brink AATP, Meijer CJLM, Wiegerinck MAHM, et al. High concordance of results of testing for human papillomavirus in cervicovaginal samples collected by two methods, with comparison of a novel self-sampling device to a conventional endocervical brush. J Clin Microbiol. 2006;44:2518-2523. doi:10.1128/ JCM.02440-05

156. Othman NH, Mohamad Zaki FH, Nik Hussain NH, Wan Yusoff WZ, Ismail P. Self-sampling versus physicians' sampling for cervical cancer screening - agreement of cytological diagnoses. Asian Pacific J Cancer Prev. 2016;17(7):3489-3494. doi:10.1016/j.pathol.2015.12.402

157. Singla AA, Komesaroff P. Self-collected Pap smears may provide an acceptable and effective method of cervical cancer screening. Health Sci Rep. 2018;1:e33. doi:10.1002/hsr2.33

158. Da Silva Rocha A, Schaeffer PG, Meurer L, Hartmann CR, Edelweiss MIA. Assessment of the fournier ${ }^{\circledR}$ cervical specimen self-sampling device using the papanicolaou method. Acta Cytol. 2012;56:520-526. doi:10.1159/000341239

159. Budge M, Halford J, Haran M, Mein J, Wright G. Comparison of a self-administered tampon ThinPrep test with conventional pap smears for cervical cytology. Aust New Zeal J Obstet Gynaecol. 2005;45:215-219. doi:10.1111/j.1479-828X.2005.00392.x
160. Jones HE, Mansukhani MM, Tong GX, Westhoff CL, Baradaran HR. Validity and reliability of using a self-lavaging device for cytology and hpv testing for cervical cancer screening: findings from a pilot study. PLoS One. 2013;8:e82115. doi:10.1371/journal.pone.0082115

161. Loopik DL, Melchers WJG, Vedder JEM, et al. Reflex cytology for triage of high-risk human papillomavirus positive self-sampled material in cervical cancer screening: a prospective cohort study. BJOG Int J Obstet Gynaecol. 2020;127 (13):1656-1663. doi:10.1111/1471-0528.16352

162. Wentzensen N, von Knebel Doeberitz M. Biomarkers in cervical cancer screening. Dis Markers. 2007;23:315-330. doi:10.1155/ 2007/678793

163. Gradíssimo A, Burk RD. Molecular tests potentially improving HPV screening and genotyping for cervical cancer prevention. Expert Rev Mol Diagn. 2017;17:379-391. doi:10.1080/ 14737159.2017.1293525

164. Fan Y, Meng Y, Yang S, et al. Screening of cervical cancer with self-collected cervical samples and next-generation sequencing. Dis Markers. 2018;2018:1-4. doi:10.1155/2018/4826547

165. Xu B, Chotewutmontri S, Wolf S, et al. Multiplex identification of human papillomavirus 16 DNA integration sites in cervical carcinomas. PLoS One. 2013;8:e66693. doi:10.1371/journal. pone.0066693

166. Bowden SJ, Kalliala I, Veroniki AA, et al. The use of human papillomavirus DNA methylation in cervical intraepithelial neoplasia: a systematic review and meta-analysis. EBioMedicine. 2019;50:246-259. doi:10.1016/j.ebiom.2019.10.053

167. Kelly H, Benavente Y, Pavon MA, De Sanjose S, Mayaud P, Lorincz AT. Performance of DNA methylation assays for detection of high-grade cervical intraepithelial neoplasia (CIN2+): a systematic review and meta-analysis. $\mathrm{Br} J$ Cancer. 2019;121:954-965. doi:10.1038/s41416-019-0593-4

168. El Aliani A, El-Abid H, El Mallali Y, Attaleb M, Ennaji MM, El Mzibri M. Association between gene promoter methylation and cervical cancer development: global distribution and a meta-analysis. Cancer Epidemiol Biomarkers Prev. 2021;30:450-459. doi:10.1158/1055-9965.EPI-20-0833

169. Chang CC, Huang RL, Liao YP, et al. Concordance analysis of methylation biomarkers detection in self-collected and physician-collected samples in cervical neoplasm. BMC Cancer. 2015;15:1-9. doi:10.1186/s12885-015-1411-x

170. Gillio-Tos A, Fiano V, Grasso C, et al. Assessment of viral methylation levels for high risk HPV types by newly designed consensus primers PCR and pyrosequencing. PLoS One. 2018;13: e0194619. doi:10.1371/journal.pone.0194619

171. De Vuyst H, Alemany L, Lacey C, et al. The burden of human papillomavirus infections and related diseases in sub-saharan Africa. Vaccine. 2013;31:F32-F46. doi:10.1016/j.vaccine.20 12.07.092

172. Onyango CG, Ogonda L, Guyah B, et al. Novel biomarkers with promising benefits for diagnosis of cervical neoplasia: a systematic review. Infect Agent Cancer. 2020;15:1-2. doi:10.1186/s13027-020-00335-2

173. Yu L, Fei L, Liu X, Pi X, Wang L, Chen S. Application of p16/ Ki-67 dual-staining cytology in cervical cancers. $J$ Cancer. 2019;10:2654-2660. doi:10.7150/jca.32743

174. Wentzensen N, Schwartz L, Zuna RE, et al. Performance of p16/ Ki-67 immunostaining to detect cervical cancer precursors in a colposcopy referral population. Clin Cancer Res. 2012;18:4154-4162. doi:10.1158/1078-0432.CCR-12-0270

175. Luttmer R, Dijkstra MG, Snijders PJF, et al. p16/Ki-67 dual-stained cytology for detecting cervical (pre)cancer in a HPV-positive gynecologic outpatient population. Mod Pathol. 2016;29:870-878. doi:10.1038/modpathol.2016.80 
176. Ebisch RMF, Van Der Horst J, Hermsen M, et al. Evaluation of p16/Ki-67 dual-stained cytology as triage test for high-risk human papillomavirus-positive women. Mod Pathol. 2017;30:1021-1031. doi:10.1038/modpathol.2017.16

177. Mandal R, Ghosh I, Banerjee D, et al. Correlation between p16/ Ki-67 expression and the grade of cervical intraepithelial neoplasias. Int $J$ Gynecol Pathol. 2020;39:384-390. doi:10.1097/PGP.0000000000000617

178. Prevodnik VK, Marinsek ZP, Zalar J, et al. Evaluation of the training program for p16/Ki-67 dual immunocytochemical staining interpretation for laboratory staff without experience in cervical cytology and immunocytochemistry. Radiol Oncol. 2020;54:201-208. doi:10.2478/raon-2020-0018

179. Ziemke P. P16/Ki-67 immunocytochemistry in gynecological cytology: limitations in practice. Acta Cytol. 2017;61:230-236. doi:10.1159/000475979

180. Toliman PJ, Phillips S, de Jong S, et al. Evaluation of p16/Ki-67 dual-stain cytology performed on self-collected vaginal and clinician-collected cervical specimens for the detection of cervical pre-cancer. Clin Microbiol Infect. 2020;26:748-752. doi:10.1016/ j.cmi.2019.10.020

181. Orang'O EO, Were E, Rode O, et al. Novel concepts in cervical cancer screening: a comparison of VIA, HPV DNA test and p16INK4a/Ki-67 dual stain cytology in Western Kenya. Infect Agent Cancer. 2020;15:1. doi:10.1186/s13027-020-00323-6

182. Derbie A, Mekonnen D, Woldeamanuel Y, Van Ostade X, Abebe T. HPV E6/E7 mRNA test for the detection of high grade cervical intraepithelial neoplasia (CIN2+): a systematic review. Infect Agent Cancer. 2020;15:1. doi:10.1186/s13027020-0278-x.

183. Macedo ACL, Gonçalves JCN, Bavaresco DV, Grande AJ, Chiaramonte Silva N, Rosa MI. Accuracy of mRNA HPV tests for triage of precursor lesions and cervical cancer: a systematic review and meta-analysis. J Oncol. 2019;2019:1-14. doi:10.1155/ 2019/6935030

184. Pardini B, De Maria D, Francavilla A, Di Gaetano C, Ronco G, Naccarati A. MicroRNAs as markers of progression in cervical cancer: a systematic review. BMC Cancer. 2018;18:1-7. doi:10.1186/s12885-018-4590-4.

185. Burger EA, Sy S, Nygard M, Kristiansen IS, Kim JJ. Prevention of HPV-related cancers in Norway: cost-effectiveness of expanding the HPV vaccination program to include pre-adolescent boys. PLoS One. 2014;9:e89974. doi:10.1371/journal.pone.0089974

186. Burger EA, Sy S, Nygard M, Kim JJ. The cost-effectiveness of cervical self-sampling to improve routine cervical cancer screening: the importance of respondent screening history and compliance. Cancer Epidemiol Biomarkers Prev. 2017;26:95-103. doi:10.1158/1055-9965.EPI-16-0350
187. Aarnio R, Östensson E, Olovsson M, Gustavsson I, Gyllensten U. Cost-effectiveness analysis of repeated self-sampling for HPV testing in primary cervical screening: a randomized study. $B M C$ Cancer. 2020;20:1-9. doi:10.1186/s12885-020-07085-9.

188. Medical Services Advisory Committee. MSAC outcomes public summary document: application no. 1276 - renewal of the National Cervical Screening Program. 2014.

189. Mwanahamuntu MH, Sahasrabuddhe VV, Stringer JSA, Parham GP. Integrating cervical cancer prevention in HIV/AIDS treatment and care programmes. Bull World Health Organ. 2008;86:D-E. doi:10.2471/blt.08.056275

190. Odafe S, Torpey K, Khamofu H, et al. Integrating cervical cancer screening with HIV care in a district hospital in Abuja, Nigeria. Niger Med J. 2013;54:176. doi:10.4103/0300-1652.114590

191. Dobrow MJ, Hagens V, Chafe R, Sullivan T, Rabeneck L. Consolidated principles for screening based on a systematic review and consensus process. CMAJ. 2018;190:E422-E429. doi:10.1503/cmaj.171154

192. Wilson JMG, Jungner G, WHO WHO. Principles and practice of screening for disease / J. M. G. Wilson, G. Jungner. Public Health Pap. Published online 1968;123(3):349. doi:10.1001/ archinte. 1969.00300130131020

193. Poljak M, Oštrbenk Valenčak A, Gimpelj Domjanič G, Xu L, Arbyn M. Commercially available molecular tests for human papillomaviruses: a global overview. Clin Microbiol Infect. 2020;26:1144-1150. doi:10.1016/j.cmi.2020.03.033

194. Hoste G, Vossaert K, Poppe WAJ. The clinical role of HPV testing in primary and secondary cervical cancer screening. Obstet Gynecol Int. 2013;2013:1-7. doi:10.1155/2013/610373

195. Denny L, de Sanjose S, Mutebi M, et al. Interventions to close the divide for women with breast and cervical cancer between low-income and middle-income countries and high-income countries. Lancet. 2017;389:861-870. doi:10.1016/S01406736(16)31795-0

196. Nath J, Bhattacharyya A, Deka H. Comparative study between pap smear and visual inspection with acetic acid (via) in screening of CIN and early cervical cancer. J Midlife Health. 2015;6:53. doi:10.4103/0976-7800.158942

197. Gravitt PE, Paul P, Katki HA, et al. Effectiveness of VIA, pap, and HPV DNA testing in a cervical cancer screening program in a Peri-Urban community in Andhra Pradesh, India. PLoS One. 2010;5:e13711. doi:10.1371/journal.pone.0013711

198. de Sanjosé S, Diaz M, Castellsagué X, et al. Worldwide prevalence and genotype distribution of cervical human papillomavirus DNA in women with normal cytology: a meta-analysis. Lancet Infect Dis. 2007;7:453-459. doi:10.1016/S1473-3099(07)70158-5

\section{Publish your work in this journal}

The International Journal of Women's Health is an international, peerreviewed open-access journal publishing original research, reports, editorials, reviews and commentaries on all aspects of women's healthcare including gynecology, obstetrics, and breast cancer. The manuscript management system is completely online and includes a very quick and fair peer-review system, which is all easy to use. Visit http://www.dovepress.com/testimonials.php to read real quotes from published authors. 